\title{
Imaging and characterizing cells using tomography
}

Myan Do ${ }^{a, b, c}$, Samuel A. Isaacson ${ }^{d}$, Gerry McDermott ${ }^{a, b, c}$, Mark A. Le Gros ${ }^{a, b, c}$, \& Carolyn A. Larabell ${ }^{a, b, c^{*}}$

${ }^{a}$ Department of Anatomy, University of California San Francisco, San Francisco, CA 94143

${ }^{\mathrm{b}}$ National Center for X-ray Tomography, Lawrence Berkeley National Laboratory, Berkeley CA 94720

${ }^{\mathrm{c}}$ Physical Biosciences Division, Lawrence Berkeley National Laboratory, Berkeley CA 94720

${ }^{\mathrm{d}}$ Department of Mathematics and Statistics, Boston University, Boston, MA 02215

* Corresponding author: Carolyn.Larabell@ucsf.edu

Keywords:

Correlated, cryogenic, fluorescence, microscopy, modeling, nucleus, soft x-ray tomography

\section{Abbreviations:}

ALS, Advanced Light Source; CCD, charge-coupled device; CLM, cryogenic confocal light microscope; CFT, cryogenic fluorescence tomography; CFT-SXT, correlated CFT and SXT; FBP, filtered back projection; GFP, green fluorescent protein; LAC, linear absorption coefficient; NCXT, National Center for X-ray Tomography; OSN, olfactory sensory neurons; SXM, soft Xray microscopy; SXT, soft $\mathrm{x}$-ray tomography; $\mathrm{Xi}$, inactive $\mathrm{X}$ chromosome; ZP, zone plate. 


\section{ABSTRACT}

We can learn much about cell function by imaging and quantifying sub-cellular structures, especially if this is done non-destructively without altering said structures. Soft x-ray tomography (SXT) is a high-resolution imaging technique for visualizing cells and their interior structure in 3D. A tomogram of the cell, reconstructed from a series of $2 \mathrm{D}$ projection images, can be easily segmented and analyzed. SXT has a very high specimen throughput compared to other high-resolution structure imaging modalities; for example, tomographic data for reconstructing an entire eukaryotic cell is acquired in a matter of minutes. SXT visualizes cells without the need for chemical fixation, dehydration, or staining of the specimen. As a result, the SXT reconstructions are close representations of cells in their native state. SXT is applicable to most cell types. The deep penetration of soft $x$-rays allows cells, even mammalian cells, to be imaged without being sectioned. Image contrast in SXT is generated by the differential attenuation soft x-ray illumination as it passes through the specimen. Accordingly, each voxel in the tomographic reconstruction has a measured linear absorption coefficient (LAC) value. LAC values are quantitative and give rise to each sub-cellular component having a characteristic LAC profile, allowing organelles to be identified and segmented from the milieu of other cell contents. In this chapter, we describe the fundamentals of SXT imaging and how this technique can answer real world questions in the study of the nucleus. We also describe the development of correlative methods for the localization of specific molecules in a SXT reconstruction. The combination of fluorescence and SXT data acquired from the same specimen produces composite 3D images, rich with detailed information on the inner workings of cells. 


\section{Introduction}

Imaging is absolutely fundamental to continued progress in cell biology. While this may sound like a bit of an overstatement, it is difficult to deny the historic and ongoing contribution of microscopy to our understanding of cells [1]. Indeed, highly magnified images of cells are the context into which all other biochemical, biophysical and genetic measurements are viewed [2]. In an ideal world, data from a single microscope would be sufficient information to build a complete picture of a cell, but in reality this is an impossible dream [3]. Each type of microscope has unique imaging characteristics and, as a result, is only capable of generating data within a well-defined window of spatial resolution and image formation mechanisms [4, 5]. To overcome this limitation a range of different, information-specific microscopes are used to image cells. For example, fluorescence microscopes are typically used to track and locate the positions of tagged molecules in the cell [6-9]. Electron microscopy, on the other hand, is best suited to generating very high spatial resolution images of the cellular ultrastructure $[10,11]$. The availability of new technologies - such as CCD cameras, laser illumination sources, genetically encodable fluorescent labels, and high-performance computers - together with the implementation of new powerful, high-performance algorithms are pushing light- and electron-based microscopies to new limits. However, electron- and light-based methods are not well suited to imaging nativestate specimens at the functionally important mesoscale (i.e. at a spatial resolution of a few tens of nanometers) $[12,13]$. The development of soft $x$-ray based methods for imaging cells filled this gap in cellular imaging [14].

Soft $x$-rays penetrate hydrated specimens more deeply than electrons do, and produce bright-field images with much higher resolution than is possible using light [15-17]. As a result, eukaryotic cells can be imaged intact, without sectioning or milling [18-21]. In soft x-ray microscopy (SXM) the image contrast is generated directly by the bio-molecular composition at 
each point in the cell $[17,22]$. There is, therefore, no need to stain cells, or treat them with heavy metals or other contrast enhancing agents prior to imaging [23]. Due to these advantages in specimen preparation, soft x-ray microscopy meets the criteria of complementing data from existing modalities whilst providing totally unique views of cells.

As with the majority of microscopes, SXMs only produce 2D projection images of the specimen [24]. The complexity of even a simple cell makes projection images difficult if not impossible to interpret, since sub-cellular structures end up confusingly superimposed in the image. To address this shortfall, tomographic methods are used to calculate 3D reconstructions of the specimen from a series of 2D SXM images [25]. Below we describe the application of transmission soft $\mathrm{x}$-ray tomography (SXT) to imaging cells. We will also discuss the development of correlative methods that can partner with SXT, specifically the recent development of cryogenic fluorescence tomography (CFT).

In correlative CFT-SXT imaging the cryopreserved specimen is imaged in a cryogenic highnumerical aperture light microscope prior to being cryo-transferred to the SXM for acquisition of SXT data [26-29]. Landscape markers, clearly visible in data from both modalities, are used to accurately overlay one data set onto the other to produce a 3D multi-modal reconstruction [26]. In such analyses, labeled-molecules are localized precisely within high-resolution reconstructions of the cell. This combination of data from two complementary modalities produces significantly greater insights than is possible using SXT alone. We confidently predict this correlative method will become an important new tool for elucidating connections between molecular events and cellular phenotype. Correlated CFT-SXT can be applied to virtually any type of cell, presenting enormous imaging opportunities to the cell bio-research community. For this manuscript we will use the eukaryotic nucleus as a working example, since imaging and quantifying this organelle in its near-native state previously proved to be very challenging using 
other modalities [30]. Using CFT-SXT the nucleus can be fully segmented, based on the degree of chromatin compaction together with guidance from localized fluorescent molecules [31]. Information on the topology and packaging of the genome in the nucleus is invaluable to the generation of mathematical models that describe, for example, the movement of transcription factors and other molecules within the packed nuclear space [32]. Finally, the CFT data is used to unambiguously identify fluorescently labeled chromosome territories, creating a perfect structural complement to high-throughput molecular biology techniques, such as chromosome conformation capture (3C) [33].

\section{Soft X-ray Microscopy}

If you have used a simple bright-field light microscope, you will already be familiar with the optical layout of a transmission soft x-ray microscope (SXM). In common with light microscopes, a SXM consists of a condenser lens, which focuses illuminating light onto the specimen, a specimen stage, and an objective lens, which focuses the light transmitted through the specimen onto a detector $[15,16,34,35]$. Typically, the detector is a thinned back-illuminated charge coupled detector (CCD) that directly measures photons transmitted by the specimen (as opposed to using a scintillator that is either bonded or fiber-coupled to the detector) [24]. Where light microscopes and SXMs differ most is in the detailed construction of the lenses. The refractive index of most materials towards soft x-rays is close to unity, making glass lenses unsuitable for use in SXM (besides, normal incidence glass lenses, such as those used in a typical light microscope, would completely attenuate the illumination). Instead, SXMs are equipped with either capillary optics and/or Fresnel zone plates (ZP) [16, 36]. ZPs are diffractive optics, nanofabricated to generate concentric rings that alternate between opaque and transparent in the soft $\mathrm{x}$-ray regime [37]. The maximum spatial resolution with which a SXM can image is generally determined by the thickness of the outermost ring in the objective lens [16, 36, 37]. 
The current generation of ZP objectives in everyday SXM use has a maximum resolution of 25 $\mathrm{nm}$, but there are also ZPs available with an outer zone width approaching $10 \mathrm{~nm}$ [38]. These very high-resolution lenses could be fitted into existing SXMs easily, since spatial resolution has no impact on the physical size of the ZP. However, as the spatial resolution increases the depth of field (DOF) decreases [39]. The DOF for a $10 \mathrm{~nm}$ objective ZP is in the region of $500 \mathrm{~nm}$ to 2 $\mu \mathrm{m}$, depending on the exact configuration of the instrument [34]. This DOF is smaller than most cells, certainly almost all eukaryotic cells. But, the limitations imposed by a shallow DOF could be overcome by collecting through-focus data at each angle in the series. Consequently, work is now underway to develop imaging acquisition and data processing strategies that permit use of enhanced resolution ZPs.

As with all microscopes SXMs require a source of illumination. In the case of a SXM this currently means a beam of soft x-ray photons produced by a synchrotron. Synchrotrons, such as the Advanced Light Source (ALS) in Berkeley, are capable of generating a continuous stream of soft $x$-rays with astronomical intensity, literally. The light typically produced by a thirdgeneration synchrotron, such as the ALS, is a billion times brighter than the sun (http://wwwals.lbl.gov/index.php/about-the-als/quick-facts.html). Synchrotrons also produce a continuum of radiation, making it possible to tune the SXM to a particular energy for data collection [24]. Whilst this feature is not yet heavily utilized, it is clear that in the future there will be a significant demand for imaging data collected with illumination above and below absorption edges, in particular the phosphorus K-edge for detailed studies of the nucleus.

The disadvantage to using a synchrotron is limited access and having to travel to a central facility to collect data, rather than being able to do so within the home institution. Consequently, laboratory scale soft x-ray sources, commonly known as 'table-top' sources, have been under intense development for a number of years [40-43]. SXT remains a synchrotron- 
based modality in the meantime, though the prospects of a suitable 'table-top' microscope becoming available in the near-term are very good.

As an example of a SXM we will now briefly describe Beamline 2.1 at the Advanced Light Source, Berkeley. For detailed specifications and a full description of this instrument see [24]. Known as XM-2, this microscope was designed and built by the National Center for X-ray Tomography (NCXT) specifically for biological imaging, in particular, cells. XM-2 utilizes a bendmagnet in the ALS storage ring lattice as the source of soft x-rays. To give an idea of scale, the complete XM-2 instrument, from the bend-magnet source to the CCD detector, is $19.71 \mathrm{~m}$ long (Fig. 1). In principle, XM-2 can be tuned to operate with monochromatic illumination energy between 400 and $1300 \mathrm{eV}$ [24]. To date, all experimental data have been collected on XM-2 using 'water window' illumination (284-543 eV), typically $520 \mathrm{eV}$. The optical set-up of XM-2 is that of a classical two-lens bright-field light microscope, with Fresnel zone plate condenser and objective lenses (Fig.2) [24]. A flat mirror between the source and the condenser acts as a cutoff filter for high-energy photons. The condenser lens, which also acts as a monochromator, creates a 40x demagnified image of the $x$-ray source in the specimen imaging region and also produces a $1 \times 1 \mu \mathrm{m}$ spot on the specimen [24]. Since this spot is smaller than most cells being imaged, it is scanned across the field of view during acquisition of a projection image. The objective lens transmits a magnified image of the illuminated specimen onto the CCD (typically the magnification is 2000x). In its current operating mode, XM-2 has a maximum spatial resolution of $35-50 \mathrm{~nm}$. With a $50-\mathrm{nm}$ zone plate and water window illumination $(520 \mathrm{eV})$ the DOF is $10 \mu \mathrm{m}$, approximately. The specimen rotation stage must be maintained at low temperature during experiments. Imaging at cryogenic temperature helps mitigate artifacts appearing in images due to radiation damage. All biological specimens experience damage when exposed to intense sources of illumination, whether this is electrons, light or soft x-rays. 
Since tomography depends on the acquisition of a series of images from an invariant specimen, it is important that the specimen does not deteriorate or change during data collection, at least not on a size scale discernible in images. In the case of SXT at cryogenic temperatures the specimen will change, but mostly at the molecular level or at a size scale too small to be detected in images.

\section{Technical considerations}

Of course, as in any endeavor in life, there is a range of different but equally applicable solutions to any technical challenge. The SXMs currently available for use by cell biologists all adhere to the same basic design concepts, but differ in execution (other SXMs are now in operation at synchrotrons in Spain (https://www.cells.es/Beamlines/XM) and Germany (http://www.helmholtz-berlin.de) and coming online in the UK at the Diamond Light Source (http://www.diamond.ac.uk/Beamlines/Imaging/B24.html)). For example, the type of synchrotron insertion device that acts as the source of illumination, condenser design, the type of specimen mount used, and the method used to maintain the specimen at cryogenic temperature. In terms of this manuscript, and its intended readership, a full discussion of these differences isn't particularly important. The attention of interested reader is, however, drawn to a couple of consequences that arise from technical differences between SXMs.

For the best possible tomographic reconstruction of the specimen, in addition to the specimen remaining invariant throughout imaging, the data should be as complete as possible. Missing data, particular systematically missing data, has a negative effect on the quality of the reconstruction [26]. This is easily understandable if we consider the tomographic process from first principles, namely the central slice theorem, i.e. the 2D Fourier Transform of an object is identical to a plane of the object's 3D Fourier transform normal to the projection direction (i.e. along the optical axis) (for a general discussion of tomographic reconstruction we highly 
recommend the recent book edited by G.T. Herman and J. Frank [44]). Because there is no depth (z) in 2D projection images this information must be computed using multiple images, for example by deconvolution in the case of images from a single perspective, or tomography if the specimen is imaged from multiple angles around a rotation axis [45-47]. By acquiring projection images over a range of angles the entire Fourier transform can be measured and a 3D reconstruction calculated. The number of images required is a function of specimen size and the spatial resolution required. The minimum number of equal spaced projections that must be collected over a total angular range $180^{\circ} \mathrm{N}=\pi \mathrm{D} / \mathrm{d}$ where $\mathrm{D}$ is the specimen diameter, and $\mathrm{d}$ is the spatial resolution [48]. Clearly, the optimum tomographic reconstruction requires these criteria be fully met.

In SXT the use of flat specimen mounting grids limits the maximum tilt angles to approximately $\pm 70^{\circ}$ [49]. As the tilt angle increases so does the thickness of the specimen with respect to illumination normal to the rotation axis [26]. Take, for example, a $5.0 \mu \mathrm{m}$ thick specimen, as described by Cinquin and colleagues [26]. When the specimen is at a $45^{\circ}$ tilt angle, the beam passes through $7.1 \mu \mathrm{m}$ and, at the common maximum tilt of $72,^{\circ}$ the beam passes through $16 \mu \mathrm{m}$; hence, the sample is 3.2 times thicker here than it is at $0^{\circ}$. Both the restricted tilt series of the flat grid and variable sample thickness have several negative consequences on the resulting image data. First, the LAC values cannot be consistently or accurately correlated to specific cellular structures. More importantly, a significant portion of the Fourier transform is not sampled because of the lack of rotation. This leads to systematically missing data, eponymously named the 'missing wedge' because of its shape. Lastly, flat-grid SXT is incompatible with cryo-fluorescence tomography (CFT), which requires a full $360^{\circ}$ rotation, preventing valuable correlation of data from CFT and SXT. The XM-2 microscope at the ALS bypasses these issues by utilizing a cryogenic rotation stage that freely rotates around a 
complete $360^{\circ}$ [25]. Specimens are mounted in very thin-walled capillary tubes, which in turn are mounted on the cryo-rotation stage [25]. However, capillary SXT cannot easily accommodate extended specimens such as adherent cells. These cell types are more readily imaged using electron microscopy style grids and associated cryo-stage [49].

\section{Calculating a 3D reconstruction of the specimen from 2D data}

Tomography is a well-established method for reconstructing the interior of an object in 3D from 2D projections [50-52]. For a full description see other chapters in this edition. In brief, the simplest method for reconstructing SXT data is the Filtered Back Projection (FBP). This method is very quick, and can calculate a reconstruction of a cell in near real time using very modest computational resources. A laptop has sufficient computational power to reconstruct a FBP reconstruction of SXT data in a reasonable timeframe. However, FBP relies on the assumption that SXT data behaves like a pure Radon transform [50]. On the other hand, iterative reconstruction methods take account of features not captured in the Radon transform and typically produce better quality reconstruction from SXT data $[25,29,53,54]$. Since SXT is very similar in concept to the medical CT (computed axial tomography) and electron tomography, software and algorithms for SXT data processing and reconstruction are readily available.

\section{Interpreting and analyzing Soft X-ray Reconstructions}

SXM illumination typically comes from within a spectral region known as the 'water window' (284-543 eV) that lies between the absorption edges of oxygen and carbon. Illumination in this region is attenuated an order of magnitude more by carbon-rich molecules, such as proteins or lipids, than by water [37]. Attenuation of the illumination by the specimen adheres to the BeerLambert law, and is therefore linear and a function of molecular species and concentration. Differences in molecular composition thereby generate contrast in SXM images of a biological 
specimen. Densely packed proteins or lipids strongly attenuate soft x-rays, whereas highly solvated regions will attenuate weakly $[55,56]$. The degree to which a biomolecule or subcellular object attenuates is quantified by the Linear Absorption Coefficient (LAC). For example, ice has a theoretical LAC value of $0.109 \mu \mathrm{m}^{-1}$, whereas a model protein with chemical composition $\mathrm{C}_{94} \mathrm{H}_{139} \mathrm{~N}_{24} \mathrm{O}_{31} \mathrm{~S}$ has a calculated LAC value of $1.35 \mu \mathrm{m}^{-1}$ [55]. In reality, the measured LAC values correspond closely with theoretical values, with the LAC for each component in the cell falling within the values for ice and dry protein.

Each voxel in the 3D reconstruction of the cell has an associated LAC value (Fig.3). The voxel LAC value directly correlates with the species and concentration of molecules in the corresponding space in the specimen $[17,56]$. For example, densely packed lipid droplets have a high concentration of carbon and relatively little water content, resulting in a LAC value of $\sim 0.7$ $\mu \mathrm{m}^{-1}$ (i.e. lipid droplets strongly attenuate the soft $\mathrm{x}$-ray illumination). Vacuoles, on the other hand, have low carbon/high water content and lower LAC values in the range $0.22 \pm 0.07 \mu \mathrm{m}^{-1}$. LAC values of specific cellular components in a single cell population, and also in different cell types across many eukaryotic species, consistently fall within a certain range. Mammalian nuclear heterochromatin typically ranges from 0.25 to $0.36 \mu \mathrm{m}^{-1}$, and euchromatin, $0.13-0.25$ $\mu \mathrm{m}^{-1}$ [33]. Similarly, the LAC of nuclear chromatin in yeast is $0.26 \pm 0.01 \mu \mathrm{m}^{-1}$ [57]. Other LAC value examples include the nucleolus, $0.33 \pm 0.01 \mu \mathrm{m}^{-1}$, and mitochondria, $0.36 \pm 0.02 \mu \mathrm{m}^{-1}$.

Fig. 3 shows a virtual section through a reconstructed cell. As can be seen from this figure, the organelles and other structures are easily distinguished by their LAC values. This characteristic makes segmentation of the cell relatively straightforward. Segmentation is the process of dividing the reconstructed cell volume into biologically relevant sub-volumes, for example isolation of particular organelles from the rest of the cell, or regions of chromatin compaction within the nucleus (Fig. 5). Some experiments require characterization of a single 
organelle, others the majority of the cell contents. Since the organelles are function-specific they tend to have a characteristic overall LAC value. The LAC values of the major organelles are not only comparable between cells of a similar strain, but between widely differing cells; for example, yeast [57] and mammalian nuclei [31] have a similar average LAC value of $0.26 \mu \mathrm{m}^{-1}$. As can be seen in Fig. 3 and 4A, differences in LAC make features such as the nuclear membrane or other organelle boundaries clear and easy to delineate. The cell is segmented (Fig. 4) using standard image analysis tools, for example IMOD [58] and Amira (http://fei.com).

\section{When is SXT the better choice for imaging cells?}

Since this paper is part of a special edition on electron microscopy - with a strong focus on 3D electron tomography - one might reasonably ask why use SXT when electron tomography is so good? To be blunt, if you need very high (better than $10 \mathrm{~nm}$ ) spatial resolution structural data your first thought should be to use an electron-based modality. However, high spatial resolution is not always a primary consideration, nor is it an absolute requirement for most questions posed in cell biology.

SXT is the better choice when the experiment requires minimally perturbed specimens, accurate visualization and quantification of whole-cell phenotype, and image data with a 25-nm maximum spatial resolution. To reiterate, the greatest advantage of SXT is imaging completely intact cells without the need to stain them with contrast-enhancing agents, due to the unique nature of soft $\mathrm{x}$-ray attenuation by biomolecules. Because soft $\mathrm{x}$-rays penetrate any given specimen up to $15 \mu \mathrm{m}$ thick, the specimen is neither sectioned nor ion-milled as is required in sample preparation for electron microscopy. SXT is also worth considering as a first choice for imaging when the experiment requires a statistically significant numbers of reconstructions. Minimal sample preparation (cryopreservation via rapid plunging into liquid propane [26]) and 
relatively quick data acquisition of a large number of cells enable SXT to be a high-throughput technique.

A recent study utilized the above advantages of SXT (and also cryo-fluorescence tomography correlated with SXT) to image the topology and overall structure of the inactive X chromosome (Xi) in female V-abl transformed thymic lymphoma cells [33]. Xi has been studied extensively for several decades using multiple microscopy techniques; however, there was previously little information on Xi's physical characteristics in nucleo due to the limitations of existing modalities. Though transmission electron microscopy (TEM) is an ideal method for the Xi ultrastructure study, it poses significant challenges. First, the nucleus must be sectioned because intact eukaryotic nuclei are far too thick to be imaged by TEM. Second, it requires artificial staining, usually with a type of heavy metal, so that image contrast is produced. These stains bind nuclear and other cellular structures nonspecifically, therefore giving little, if any, indication of biochemical composition in the cell. TEM sample preparation procedures, such as staining and detergent extraction of nucleoplasm, are also likely to disturb the in nucleo chromatin structure. Ultimately, it is impossible to image $\mathrm{Xi}$ in a near native state using TEM. SXT addresses and eliminates both aforementioned challenges. Soft $\mathrm{x}$-rays penetrate the whole cell so that sectioning is unnecessary, and are attenuated by biomolecules in a fashion that generate inherent and quantifiable image contrast.

\section{SXT in practice}

SXT has now been applied to imaging a wide range of different cell types, ranging in size from small bacteria to mammalian cells derived from living tissue. Among high-resolution structure imaging methods SXT has the unique capacity to image the nucleus in intact mammalian cells. 
The nucleus is arguably the most interesting and functionally complex of the organelles. Although the genome is defined by its sequence, how it functions is heavily controlled by its 3 dimensional topology $[31,33,30]$. The linear sequence of nucleotides in a genome is insufficient information to predict gene expression levels in cellulo. Light and electron microscopy studies on genome organization have shown the nucleus is organized spatially into functionally distinct territories. The specific spatial arrangement of genes and chromosomes in the interphase nucleus is, therefore, widely accepted as having regulatory control over gene function. Consequently, the topological arrangement adopted by the genome is key information, and essential to understanding the fundamental processes that take place in the nucleus. Moreover, imaging the nucleus together with the cellular phenotype is especially informative in the study of diseases such as cancer, where changes in nuclear shape and composition are accompanied by aberrant cell behavior.

Recent work imaging by the Lomvardas group highlighted the utility of SXT in terms of imaging the nucleus [31]. The cells imaged in this study were olfactory sensory neurons (OSNs) obtained by the dissociation of tissues from the noses of mice. The research goal was to investigate the mechanism that results in an olfactory neuron having a single olfactory receptor when several thousand olfactory receptor (OR) genes are available. The initial choice of which gene will be expressed by the neuron is likely a matter of chance, but the most pressing question is how does the cell silence the other genes? Using mutant mice, the team discovered the answer lies in the spatial arrangement of the chromosomes. The silenced OR genes were found to be in loci near the center of the nucleus, whereas the sole OR gene being expressed was located in transcriptionally active euchromatin territories near the periphery of the nucleus. This observation was tested by creating a genetically engineered mouse in which the normally silenced OR genes were fully expressed. By utilizing SXT, a dramatic change in nuclear 
organization between this mouse and the control mouse was shown [31] (Fig.5). This observation had not been possible using other modalities.

\section{Confocal cryo-tomography for correlation with SXT data}

Whilst SXT reconstructions contain significant amounts of structural information, they lack positional information on key molecules. Identifying the location of particular molecules within the cell is essential in many research projects. Proteins can be identified directly in a SXT reconstruction by labeling the molecule of interest with an electron dense tag, for example goldnanoparticles conjugated to an antibody. However, getting the gold-nanoparticle inside the cell requires chemically fixing and then permeabilizing the cell, a process that alters the in vivo structure and molecular organization. Alternatively, tagging molecules of interest with a fluorescent label, for example Green Fluorescent Protein (GFP), does not alter or damage the organization or structure of the cell. As such, fluorescent tagging is the preferred localization method in most cases. Fluorescence microscopy is a generally non-invasive technique that has become standard practice in virtually every cell biology lab.

The simplest way to combine SXT and fluorescence is to image the fluorescence signal, and then rapidly cryo-preserve the specimen. Whilst basic and easy to achieve the outcome of the experiment is the potential combination of two non-identical specimens. Even though the specimen is fixed very soon after fluorescence imaging, this time lag is sufficient for changes in cell structure and molecular organization to occur. The more desirable method is to cryopreserve the cell prior to imaging by either modality (as an aside, fluorescence data is always collected first since SXT acquisition damages fluorophores [49]).

Low-temperature light microscopes have been available for many years. However, these instruments have typically been fitted with low numerical aperture lenses. As a result, the 
specimen and the objective are coupled by air. Whilst these systems work, they were limited to using low numerical aperture lenses, resulting in poor spatial resolution and image quality due to light passing through substances (glass lens - air - specimen) with different refractive indices. The resulting reflection and refraction of the illumination degrades image quality and generates aberrations. The development of a high-numerical aperture cryogenic microscope allowed the specimen to be coupled to the objective via a refractive index matched fluid, typically propane at 77K [59]. By fitting a rotation stage to the cryo-light microscope, data for cryogenic fluorescence tomographic data can be collected (Fig. 6) [26]. The reason for taking a tomographic approach to fluorescence data acquisition is simple. The cryo-light microscope produces data with very good spatial resolution in $\mathrm{x}$ and $\mathrm{y}$, but poor resolution along the optical axis (z), even if a through-focus images series is collected and deconvolved. Sequentially collecting through-focus data sets at different angular perspectives around a rotation axis improves the resolution of the fluorescent signal along the optical axis, and ultimately matches that measured in $x$ and $y$. Achieving anisotropy in molecular localization is important for the accuracy of the overlay of fluorescence data with the SXT reconstruction.

\section{CFT-SXT in practice}

Fig. 7 describes the overall process of combining CFT and SXT data. In this mode of operation the specimen is mounted in a thin-walled capillary tube. The glass wall is close in refractive index to the immersion fluid, resulting in minimal reflections of aberrations in images - the glass simply 'disappears' and the illumination and fluorescence signal pass through unimpeded. Fiducial markers are an important factor in this correlated imaging method, and judiciously chosen based on their visibility in both modalities. Having common landscape features in both data sets permits accurate co-alignment of the two 3D reconstructions using 
standard methods, such as affine transformations (Fig. 8). An example of CFT-SXT in practice is shown in Fig. 9. In this work, Smith and colleagues identified the inactive $\mathrm{X}$ chromosome (Xi) using EGFP signal (EGFP was tagged onto MacroH2A, a protein enriched on $\mathrm{Xi}$ ). By overlaying the CFT information on Xi in SXT reconstructions from the same cell it was possible to identify and segment Xi from the surrounding chromatin in the nucleus. Once segmented, Xi could be comprehensively analyzed in terms of LAC values, topology, etc. Again, the information produced by this study could not have been easily replicated using any other technique or combination of techniques. This, to our knowledge, is the first quantitative, structural view of a chromosome in an intact, near-native state cell.

\section{Using SXT reconstructions to model molecular mobility in the nucleus}

Mathematical models of biochemical systems within individual cells are widely used to help answer questions about nuclear processes. Questions include how transcription factors are distributed within nuclei [60]; what are the sources of noise in transcription [61]; and how proteins search for specific DNA binding sites [32, 61-64]. Many existing models are non-spatial, treating the nucleus as a single well-mixed volume. While non-spatial models have been very successful in approximating, predicting and explaining many nuclear processes, SXT imaging clearly demonstrates the spatial non-uniformity of material within nuclei. An active area of research is to understand how this spatial heterogeneity might influence the dynamics of nuclear processes. A key component in developing more detailed mathematical models to investigate this question is the availability of high-resolution imaging data of cellular substructure. SXT can provide large quantities of such data, including the spatial distribution of chromatin with nuclei; the location and shape of organelles and membranes; and estimates on the amount of organic material present at different locations. 
We illustrate a way that SXT reconstructions provide information needed for more accurate and detailed mathematical models, by describing the problem of how a protein searches for specific DNA binding sites. Since the classic paper of Berg et al. [63], investigating the interplay between 3D diffusion in the nucleus and 1D diffusion along DNA, there have been a large number of experimental and modeling studies investigating this question (see the many references in [64]). Currently we are investigating a different aspect of this question: how volume exclusion due to the spatially varying density of chromatin within the nucleus can influence the search process $[32,62]$. SXT reconstructions were used in [32] to determine the spatial variation in chromatin density within mouse olfactory sensory neuron nuclei. Volume exclusion due to the presence of chromatin was then modeled by constructing an effective potential field, proportional to the chromatin density, that hinders a diffusing protein from entering regions of heterochromatin (which has high density). The constant of proportionality, which we call the volume exclusivity of chromatin, is a model parameter that we vary to study the influence of volume exclusion on the random time required for a diffusing protein to find its target binding site. When this constant is zero there is no volume exclusion due to chromatin within the model. Increasing the constant then increases the strength of volume exclusion due to regions of high chromatin density. A typical path for a diffusing protein experiencing the potential while searching for a specific binding site is shown in Fig. 10B. In Fig. 10A we show a snapshot of the search process, rendering the heterochromatin within the nucleus, the position of the binding site, and the position of the protein at a fixed point in time.

Consider the search for randomly chosen binding sites located in euchromatin, which are regions of low chromatin density. We compute the median time to find such a binding site by a protein that enters the nucleus at a randomly chosen nuclear pore. As the volume exclusivity of chromatin increases from zero, we find that the median time needed to reach a target-binding 
site at first decreases to a minimum, and then increases again as the volume exclusivity of chromatin increases further. This relationship is illustrated in Fig. 10C. A theoretical analysis suggests that the observed minimal search time is obtained from a balance between reducing the effective volume within the nucleus the protein searches, with increasing the difficulty for the protein to travel between regions of euchromatin separated by heterochromatin [32]. These results suggest that chromatin could be distributed so as to help funnel proteins towards active binding sites in regions of euchromatin.

\section{Conclusions and Future Outlook}

SXT has emerged as a capable new technique for imaging and quantifying cells at the mesoscale. During the past decade enormous progress has been made. SXT has gone from being a rather esoteric technique in development by synchrotron scientists to an easy-to-use microscopy method used by cell biologists. This transition has coincided with a growth in the number of synchrotron-based SXMs worldwide, and given further impetus to the development of 'table top' soft $x$-ray sources.

The ability of SXT to provide whole-cell images, quantitative measurements related to the density of organic material, and datasets of multiple cells will be an important resource for mathematical modelers. In addition to allowing for more detailed, accurate models of nuclear

processes, SXT will offer modelers a means to create reconstructions of cytoplasmic ultrastructure (such as mitochondrial membrane locations). Such features are an important component in the development of realistic, three-dimensional models for the dynamics of chemical processes within cells.

The local cellular environment plays a significant role in controlling how and when molecules interact. Consequently, understanding cell behavior requires knowledge of both the 
sub-cellular location of particular molecules, together with a quantitative and detailed structural characterization of that specific location and the rest of the cell. The combination of CFT and SXT addresses a need for imaging technologies that complement molecular localization data with structural information. We predict this combination of modalities will be a significant presence in the field and that CFT, or some close variant, will be adopted both as a standalone technique and as a correlative partner with electron microscopy/tomography, especially as cryogenic microscopes capable of 'super resolution' imaging become available. Overall, we have to conclude the future looks very good for CFT-SXT, with the most exciting applications and results yet to come.

\section{Acknowledgements}

This work was supported by the US Department of Energy, Office of Biological and Environmental Research (DE-AC02-05CH11231), the National Center for Research Resources of the National Institutes of Health (P41RR019664), the National Institutes of General Medicine of the National Institutes of Health (GM63948), and the Gordon and Betty Moore Foundation (3497). S.A.I. was supported by NSF DMS-0920886 and NSF DMS-1255408. S.A.I. also thanks Dr. Larabell and The National Center for X-ray Tomography for hosting him during his fall 2014 sabbatical.

\section{Figure Captions}

Fig. 1. Schematic of XM-2, a transmission soft $\mathrm{x}$-ray microscope located at the Advanced Light Source, Berkeley. A bend-magnet in the synchrotron lattice generates an intense beam of radiation, which is transmitted to a flat plane mirror in the section of the instrument known as the branchline. The radiation then passes into the microscope room (this part of the instrument 
is termed the end station in synchrotron jargon). The condenser zone plate, in conjunction with a pinhole, acts as both a monochromator (to remove higher order radiation) and a focusing optic. The monochromatic illumination is focused to a $1 \times 1 \mu \mathrm{m}$ spot. To accommodate specimens larger than this spot size the condenser zone plate is scanned across the field of view during data collection. The specimen is mounted on a cryogenic rotation stage and cooled by a stream of cryogenic temperature gas at atmospheric pressure. Transmitted illumination from the specimen is focused onto a CCD detector by an objective zone plate.

Fig. 2. The SXT workflow on XM-2. Cells in suspension are transferred by pipette to a thinwalled capillary tube and rapidly cryo-immobilized. The capillary is either cryo-transferred to a storage Dewar or directly to the microscope, where it is mounted in the cryo-stage. The most significant components of XM-2 are shown schematically, namely the bend magnet source of soft x-rays and the major optical elements - mirror, condenser and objective - together with the CCD that detects illumination transmitted by the specimen. A projection image series is collected at angular increments around a central axis (typically 180 images at 1 degree rotational increments). The projection series is aligned with respect to the axis and a 3D volumetric reconstruction calculated. The reconstructed cell is segmented and quantified to provide the necessary information.

Fig. 3. A thin slice (orthoslice) through a 3D SXT reconstruction of B-cell (B lymphocyte) demonstrating the range of linear absorption coefficients (LAC) measured in a mammalian cell. Arrowhead points to example of the most absorbing organelles (lipid droplet). A caret points to the nuclear boundary, an asterisk to the nucleoli, and an arrow to a section of endoplasmic reticulum. Within the nucleus, euchromatin and the other nuclear components can be easily 
discerned from heterochromatin by their respective LAC values. Heterochromatin typically has LAC values in the range $0.25-0.36 \mu \mathrm{m}^{-1}$, compared to $0.13-0.24 \mu \mathrm{m}^{-1}$ for the remainder of the nucleus.

Fig. 4. Example of a segmented reconstruction. A) Shows the relationship between the calculated reconstruction - shown as single orthoslice - and segmented organelles. The nuclear surface is rendered in yellow. The surface of the mitochondrial network is colored gold. B) Same view as shown in (A) minus the orthoslice. All features visible in the reconstruction can be segmented and assigned an identity. The identity may be obvious - as is the case with major, well-characterized structures such as nucleus, mitochondria, nucleoli, etc. - or require additional information, such as labeling of particular molecules with known loci, to confirm identity. The segmentation of organelles is done automatically, semi-automatically or manually.

Fig. 5. A) SXT reconstruction of the control olfactory sensory neuron (OSN). The nucleus was segmented from the tomographic reconstruction using the 3D linear absorption coefficient (LAC). It is shown using a transparent surface view to reveal the chromatin. To aid visualization, the opacity and color of the obtained surface were mapped to a 3D color field with the same dimensions as the whole-cell data set. The color field and color map were chosen to highlight the condensed chromatin in the center of the nucleus. The most condensed chromatin appears dense brown with low transparency and the remaining chromatin is gray with a high degree of transparency. B) SXT reconstruction of a genetically engineered version of the OSN cells shown in (A). In this cell the olfactory receptor genes (OR), which would normally be silenced in the control mouse, were instead fully expressed. SXT visualized the dramatic change in nuclear organization between the engineered and control versions of the OSN. Figure taken from [31]. 
Fig. 6. The workflow followed to collect and reconstruct a CFT data set. Cells containing fluorescently labeled molecules are pipetted into a thin-walled glass capillary and cryoimmobilized (as in Fig. 2). The capillary is either cryo-transferred to a Dewar for storage or directly to the CLM where it is mounted in the cryo-rotation stage. Through-focus data are collected at a number of different angular rotations around a central axis (typically every $10^{\circ}$ over a total angular range of $180^{\circ}$ ). A deconvolved image is calculated for through-focus series, followed by a tomographic reconstruction of the entire series to generate a 3D fluorescence image. The use of tomographic methods greatly improves data quality, in particular by reducing anisotropy in the precision with which molecules can be localized.

Fig. 7. A correlated CFT-SXT experiment. Cells mounted in capillaries are first imaged by cryogenic fluorescence (deconvolution plus tomography). The capillary is cryo-transferred to the SXM for tomographic data collection (SXT). The two data sets are reconstructed independently before being co-aligned using common landscape markers visible in both modalities. The fluorescence overlay aids segmentation - in this case the inactive $X$ chromosome - from the remaining nuclear contents. Figure based on work described in [33].

Fig. 8. How fiducial markers are applied to CFT-SXT. The specimen is mounted in a capillary decorated with two kinds of fiducial markers: $100 \mathrm{~nm}$ diameter gold beads for the soft $\mathrm{x}$-ray data set reconstruction and $200 \mathrm{~nm}$ far-red fluorescent beads for the co-alignment of the two data sets. The fluorescent fiducials are visible in both soft x-ray and fluorescence data. The gold fiducials are only visible in SXM data. 
CFT data is collected prior to the specimen being cryo-transferred to the SXM for tomographic data acquisition. Alignment of the two data sets requires two independent reconstructions. Matching of the separate data sets is then guided by the co-alignment of the fluorescence bead sets. Figure taken from [26].

Fig. 9. An example of correlated CFT-SXT (described in detail in [33]). In this example, female mouse v-abl transformed thymic lymphoma cells transfected with EGFP-labeled histone variant macro- $\mathrm{H} 2 \mathrm{~A}$ were imaged in the cryo-light microscope. Macro- $\mathrm{H} 2 \mathrm{~A}$ is a high confidence marker for the inactive $X$ chromosome (Xi). A) Left column: Four virtual sections from the deconvolved CFT reconstruction. Middle Column: The corresponding virtual sections from the SXT reconstruction. White arrow shows the closest contact between Xi and the nucleolus. Right Column: The same sections in the combined CFT-SXT reconstruction. B) A 2-D projection of the Xi CFT reconstruction. C) Cutaway of a volume rendered SXT reconstruction. The surface of the cell is colored light blue. LAC values are represented in gray scale, ranging from high (dark) to low (light). D) The CFT reconstruction shown in (B) overlaid into the volume rendered SXT reconstruction shown in (C). E) Surface rendering of the inactive $X$ chromosome segmented from the SXT reconstruction after identification by macroH2A-EGFP CFT. The deep blue shaded areas are regions of high LAC that contact the nuclear envelope. Figure taken from [33].

Fig. 10. A) This figure shows a protein searching for a target DNA binding site inside the nucleus of a mouse olfactory sensory neuron. The protein is represented by the blue glyph while the binding site is represented by the red glyph. The gray "cloud" shows the regions of highest DNA density, as reconstructed from soft x-ray tomography images, and how they fill space within the mouse nucleus [32]. B) A typical path of a diffusing protein searching for a specific 
DNA binding site, while experiencing a volume exclusion potential due to regions of high chromatin density [32]. The green curve gives the protein's position every 0.01 seconds. The gray volume rendering shows the density of organic material at each point in space. Regions of higher density are lighter in color, while regions of lower density are darker. For this simulation heterochromatin was assumed moderately volume excluding, and hence the protein's path is generally restricted to regions of euchromatin (i.e. darker regions of low density). C) Median binding time as the strength of volume exclusion due to chromatin is increased for binding sites randomly located in euchromatin (i.e. regions of low chromatin density). Each curve corresponds to a different cell. The black curve uses DAPI stain intensity to model chromatin density [62]; the others, linear absorption coefficients from X-ray tomography reconstructions [32]. All cells exhibit a 25 to $40 \%$ reduction in binding time for moderate levels of volume exclusion. 


\section{References}

[1] P. Mazzarello, Nat. Cell Biol. 1 (1999) E13-E15.

[2] V. Roukos, T. Misteli, Histochem. Cell Biol. 142 (2014) 125-131.

[3] J.A.G. Briggs, M. Lakadamyali, Mol. Biol. Cell 23 (2012) 979-980.

[4] G. McDermott, M.A. Le Gros, C.A. Larabell, Annu. Rev. Phys. Chem. 63 (2012) 225-239.

[5] M.H. Ellisman, T.J. Deerinck, X. Shu, G.E. Sosinsky, Methods Cell Biol. 111 (2012) 139-155.

[6] S.W. Hell, Nat. Biotechnol. 21 (2003) 1347-1355.

[7] R.Y. Tsien, FEBS Lett. 579 (2005) 927-932.

[8] N.C. Shaner, P.A. Steinbach, R.Y. Tsien, Nat. Methods 2 (2005) 905-909.

[9] R.Y. Tsien, Nat. Rev. Mol. Cell Biol. Suppl (2003) SS16-21.

[10] V. Luçic, F. Forster, W. Baumeister, Annu. Rev. Biochem 74 (2005) 833-865.

[11] A. Leis, L. Andrees, M. Gruska, A. Al-Amoudi, A. Sartori, J. Dubochet, W. Baumeister, Microsc. Microanal. 11 (2005).

[12] S. Nickell, F. Beck, S.H. Scheres, A. Korinek, F. Forster, K. Lasker, O. Mihalache, N. Sun, I. Nagy, A. Sali, J.M. Plitzko, J.M. Carazo, M. Mann, W. Baumeister, Proc. Natl. Acad. Sci. U. S. A. 106 (2009) 11943-11947.

[13] S. Subramaniam, Curr. Opin. Microbiol. 8 (2005) 316-322.

[14] G. McDermott, D.M. Fox, L. Epperly, M. Wetzler, A.E. Barron, M.A. Le Gros, C.A. Larabell, Bioessays 34 (2012) 320-327.

[15] G. Denbeaux, E. Anderson, W. Chao, T. Eimuller, L. Johnson, M. Kohler, C. Larabell, M. Legros, P. Fischer, A. Pearson, G. Schultz, D. Yager, D. Attwood, Nuclear Instruments \& Methods in Physics Research Section a-Accelerators Spectrometers Detectors and Associated Equipment 467 (2001) 841-844.

[16] R. Falcone, C. Jacobsen, J. Kirz, S. Marchesini, D. Shapiro, J. Spence, Contemporary Physics 52 (2011) 293-318. 
[17] D. Weiss, G. Schneider, B. Niemann, P. Guttmann, D. Rudolph, G. Schmahl, Ultramicroscopy 84 (2000) 185-197.

[18] R. Carzaniga, M.C. Domart, L.M. Collinson, E. Duke, Protoplasma 251 (2014) 449-458.

[19] E.M. Duke, M. Razi, A. Weston, P. Guttmann, S. Werner, K. Henzler, G. Schneider, S.A. Tooze, L.M. Collinson, Ultramicroscopy 143 (2014) 77-87.

[20] A. Patwardhan, A. Ashton, R. Brandt, S. Butcher, R. Carzaniga, W. Chiu, L. Collinson, P. Doux, E. Duke, M.H. Ellisman, E. Franken, K. Grunewald, J.K. Heriche, A. Koster, W. Kuhlbrandt, I. Lagerstedt, C. Larabell, C.L. Lawson, H.R. Saibil, E. Sanz-Garcia, S. Subramaniam, P. Verkade, J.R. Swedlow, G.J. Kleywegt, Nat. Struct. Mol. Biol. 21 (2014) 841-845.

[21] C.A. Larabell, K.A. Nugent, Curr. Opin. Struct. Biol. 20 (2010) 623-631.

[22] D.Y. Parkinson, G. McDermott, L.D. Etkin, M.A. Le Gros, C.A. Larabell, J. Struct. Biol. 162 (2008) 380-386.

[23] M.A. Le Gros, C.G. Knoechel, M. Uchida, D.Y. Parkinson, G. McDermott, C.A. Larabell, in: H.E. Edward (Ed.), Comprehensive Biophysics, Elsevier, Amsterdam, 2012, pp. 90-110.

[24] M.A. Le Gros, G. McDermott, B.P. Cinquin, E.A. Smith, M. Do, W.L. Chao, P.P. Naulleau, C.A. Larabell, Journal of synchrotron radiation 21 (2014) 1370-1377.

[25] D.Y. Parkinson, L.R. Epperly, G. McDermott, M.A. Le Gros, R.M. Boudreau, C.A. Larabell, Methods Mol. Biol. 950 (2013) 457-481.

[26] B.P. Cinquin, M. Do, G. McDermott, A.D. Walters, M. Myllys, E.A. Smith, O. Cohen-Fix, M.A. Le Gros, C.A. Larabell, J. Cell. Biochem. 115 (2014) 209-216.

[27] E.A. Smith, B.P. Cinquin, M. Do, G. McDermott, M.A. Le Gros, C.A. Larabell, Ultramicroscopy 143 (2014) 33-40.

[28] E.A. Smith, B.P. Cinquin, G. McDermott, M.A. Le Gros, C.A. Larabell, in: G.C. Howard, W.E. Brown, M. Auer (Eds.), Imaging Life: Biological Systems from Atoms to TIssues, Oxford University Press, USA, 2014.

[29] E.A. Smith, B.P. Cinquin, G. McDermott, M.A. Le Gros, D.Y. Parkinson, H.T. Kim, C.A. Larabell, J. Struct. Biol. 184 (2013) 12-20.

[30] K. Luger, M.L. Dechassa, D.J. Tremethick, Nat. Rev. Mol. Cell Biol. 13 (2012) 436-447. 
[31] E.J. Clowney, M.A. Legros, C.P. Mosley, F.G. Clowney, E.C. Markenskoff-Papadimitriou, M. Myllys, G. Barnea, C.A. Larabell, S. Lomvardas, Cell 151 (2012) 724-737.

[32] S.A. Isaacson, C.A. Larabell, M.A. Le Gros, D.M. McQueen, C.S. Peskin, Bull. Math. Biol. 75 (2013) 2093-2117.

[33] Elizabeth A. Smith, G. McDermott, M. Do, K. Leung, B. Panning, Mark A. Le Gros, Carolyn A. Larabell, Biophys. J. 107 (2014) 1988-1996.

[34] D. Attwood, W. Chao, E. Anderson, J.A. Liddle, B. Harteneck, P. Fischer, G. Schneider, M. Legros, C. Larabell, Journal of Biomedical Nanotechnology 2 (2006) 75-78.

[35] M.A. Le Gros, G. McDermott, C.A. Larabell, Curr. Opin. Struct. Biol. 15 (2005) 593-600.

[36] J. Kirz, C. Jacobsen, M. Howells, Q. Rev. Biophys. 28 (1995) 33-130.

[37] D.T. Attwood, Soft X-rays and Extreme Ultraviolet Radiation: Principles and Applications, Cambridge Uhiversity Press, Cambridge, New York, 1999.

[38] W.L. Chao, B.D. Harteneck, J.A. Liddle, E.H. Anderson, D.T. Attwood, Nature 435 (2005) 1210-1213.

[39] A. Sakdinawat, Y. Liu, Opt. Lett. 32 (2007) 2635-2637.

[40] M. Berglund, L. Rymell, H.M. Hertz, T. Wilhein, Rev. Sci. Instrum. 69 (1998) 2362-2364.

[41] M. Bertilson, O. Von Hofsten, M. Lindblom, T. Wilhein, H. Hertz, U. Vogt, Appl. Phys. Lett. 92 (2008) 064104.

[42] H.M. Hertz, O. von Hofsten, M. Bertilson, U. Vogt, A. Holmberg, J. Reinspach, D. Martz, M. Selin, A.E. Christakou, J. Jerlström-Hultqvist, S. Svärd, J. Struct. Biol. 177 (2012) 267-272.

[43] H. Legall, G. Blobel, H. Stiel, W. Sandner, C. Seim, P. Takman, D.H. Martz, M. Selin, U. Vogt, H.M. Hertz, D. Esser, H. Sipma, J. Luttmann, M. Hofer, H.D. Hoffmann, S. Yulin, T. Feigl, S. Rehbein, P. Guttmann, G. Schneider, U. Wiesemann, M. Wirtz, W. Diete, Opt. Express 20 (2012) 18362-18369.

[44] Computational Methods for Three-Dimensional Reconstructions, Springer-Birkhauser, Boston, 2014.

[45] W. Baumeister, R. Grimm, J. Walz, Trends Cell Biol. 9 (1999) 81-85.

[46] F. Natterer, The Mathematics of Computerized Tomography, Wiley, New York, NY, 1986. 
[47] F. Natterer, F. Wübbeling, Mathematical Methods in Image Reconstruction, Cambridge University Press, Cambridge, New York, 2001.

[48] D. Weiss, G. Schneider, S. Vogt, P. Guttmann, B. Niemann, D. Rudolph, G. Schmahl, Nuclear Instruments \& Methods in Physics Research Section A-Accelerators Spectrometers Detectors \& Associated Equipment 467 (2001) 1308-1311.

[49] C. Hagen, P. Guttmann, B. Klupp, S. Werner, S. Rehbein, T.C. Mettenleiter, G. Schneider, K. Grunewald, J. Struct. Biol. 177 (2012) 193-201.

[50] J. Radon, Math. Phys. Klasse 69 (1917) 262-277.

[51] D.J. Derosier, A. Klug, Nature 217 (1968) 130-\&.

[52] R.A. Crowther, D.J. Derosier, A. Klug, Proceedings of the Royal Society of London Series aMathematical and Physical Sciences 317 (1970) 319-\&.

[53] D. Wolf, A. Lubk, H. Lichte, Ultramicroscopy 136 (2014) 15-25.

[54] D.Y. Parkinson, C. Knoechel, C. Yang, C.A. Larabell, M.A. Le Gros, J. Struct. Biol. 177 (2012) 259-266.

[55] D. Weiss, G. Schneider, B. Niemann, P. Guttmann, D. Rudolph, G. Schmahl, Ultramicroscopy 84 (2000) 185-197.

[56] C.A. Larabell, M.A. Le Gros, Biophys. J. 86 (2004) 185A-185A.

[57] M. Uchida, Y. Sun, G. McDermott, C. Knoechel, M.A. Le Gros, D. Parkinson, D.G. Drubin, C.A. Larabell, Yeast 28 (2011) 227-236.

[58] J.R. Kremer, D.N. Mastronarde, J.R. McIntosh, J. Struct. Biol. 116 (1996) 71-76.

[59] M.A. Le Gros, G. McDermott, M. Uchida, C.G. Knoechel, C.A. Larabell, J. Microsc. 235 (2009) 1-8.

[60] T.E. KuhIman, E.C. Cox, Mol. Syst. Biol. 8 (2012) 610.

[61] J.M. Raser, E.K. O'Shea, Science 309 (2005) 2010-2013.

[62] S.A. Isaacson, D.M. McQueen, C.S. Peskin, Proc. Natl. Acad. Sci. U. S. A. 108 (2011) 38153820. 
[63] O.G. Berg, R.B. Winter, P.H. von Hippel, Biochemistry 20 (1981) 6929-6948.

[64] S.E. Halford, Biochem. Soc. Trans. 37 (2009) 343-348. 

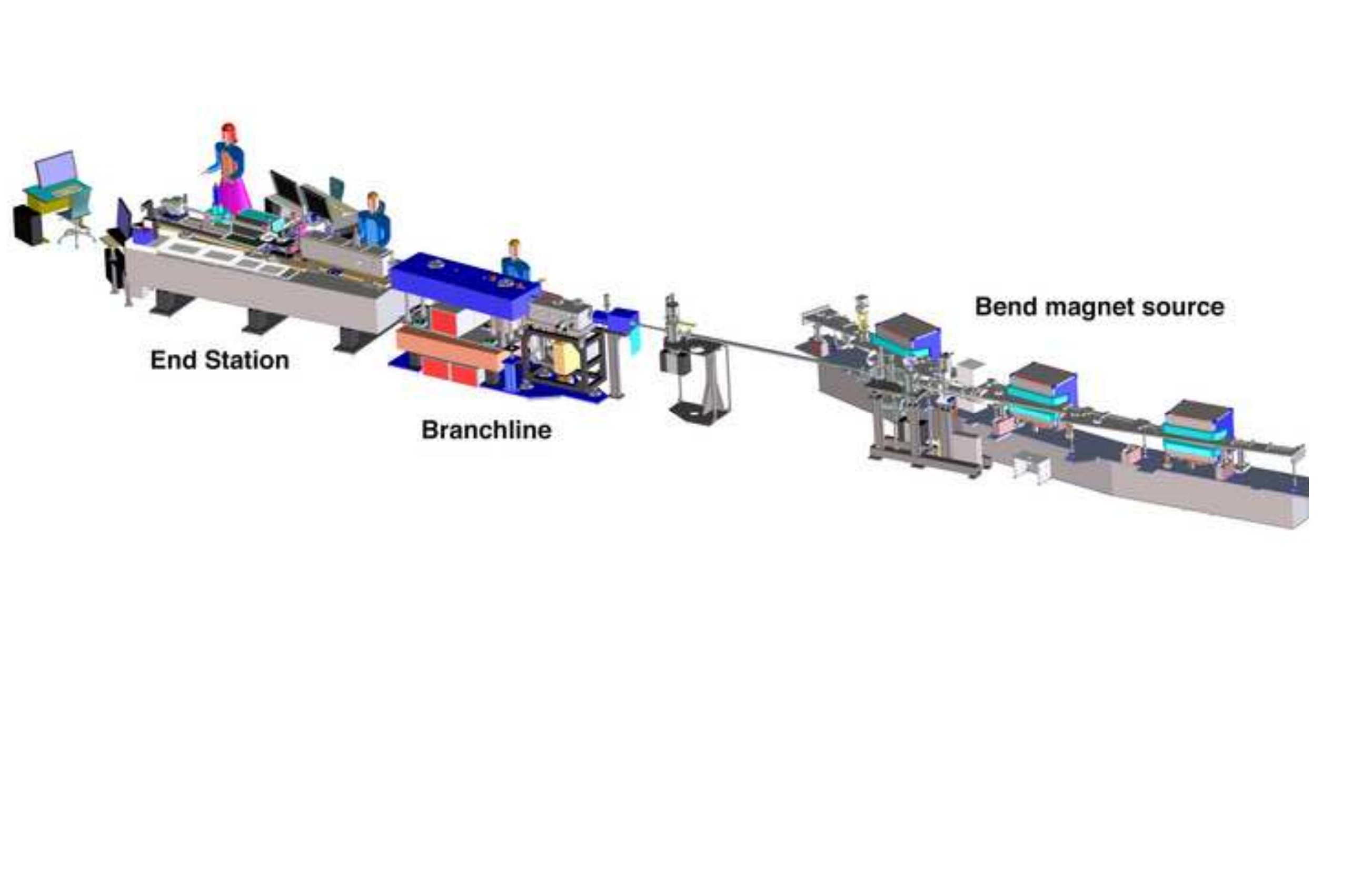

( 

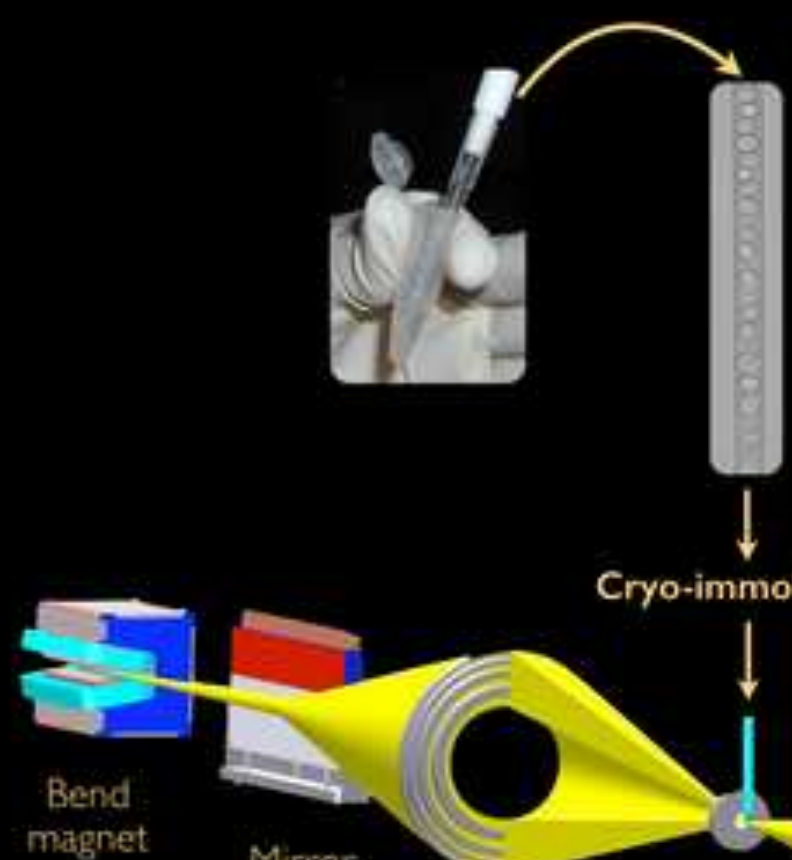

magnet
Cryo-immobilize

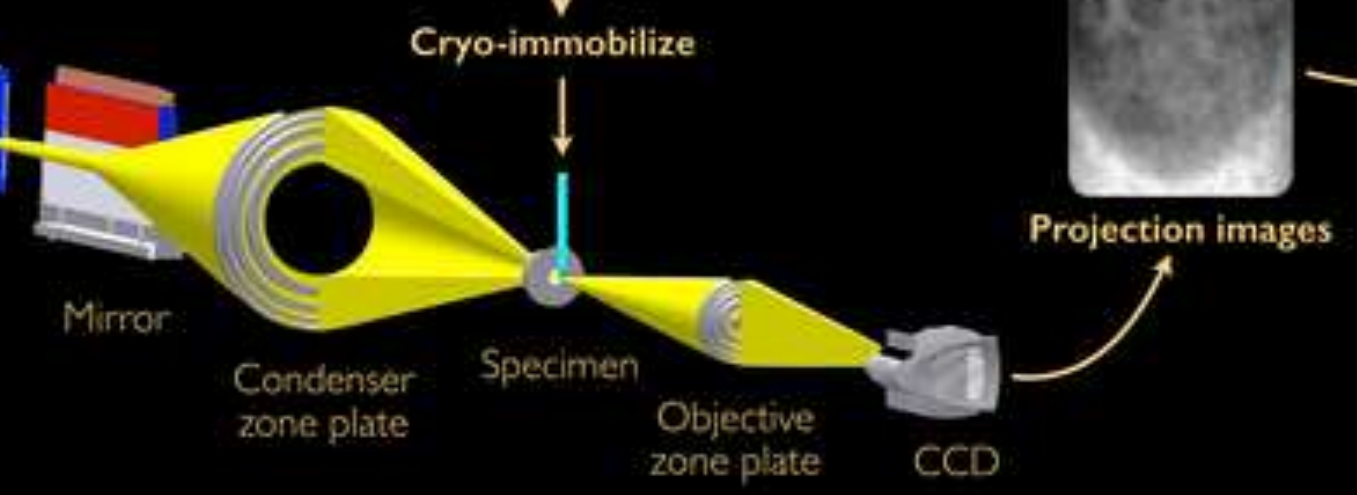

Reconstruction algorithm
Segmentation
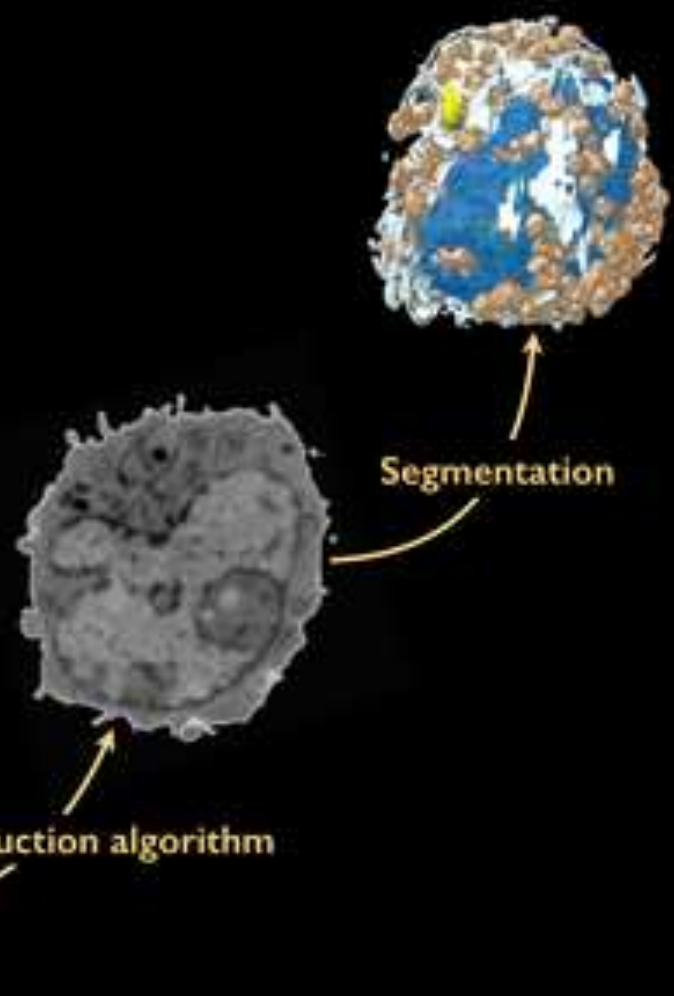
LAC Values $\left(\mu \mathrm{m}^{-1}\right)$

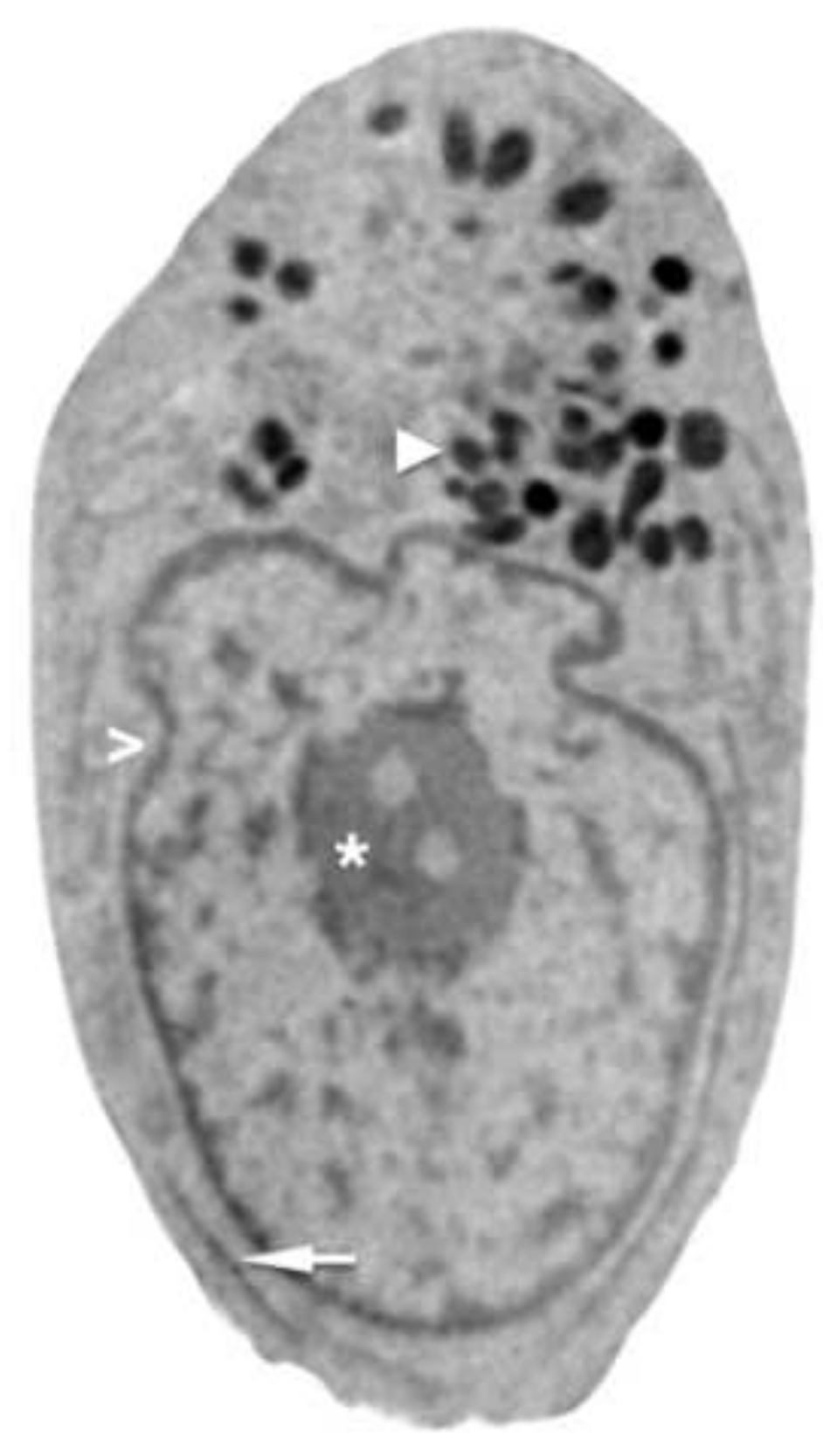

0.75

0.50

0.25

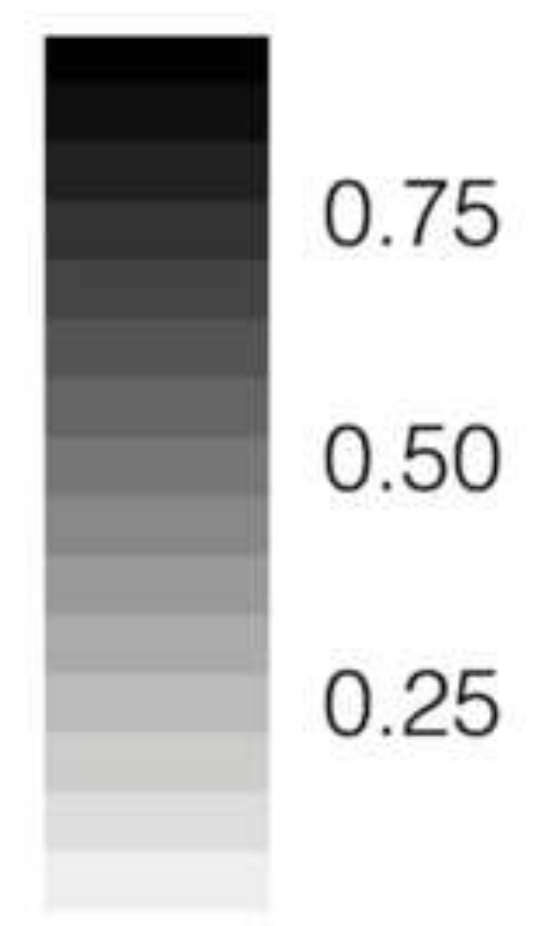



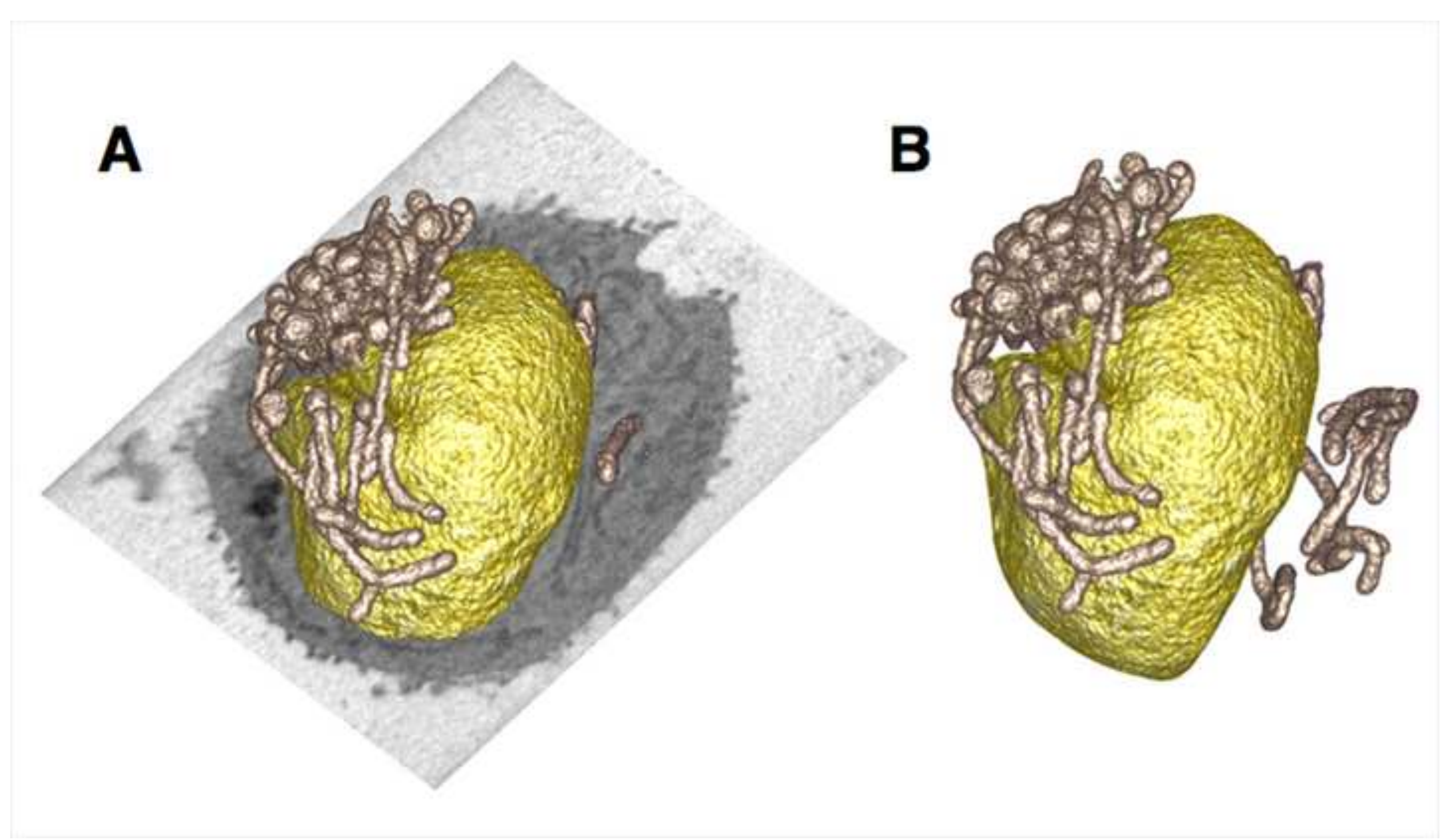

B

列

$+$

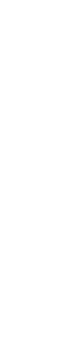


A
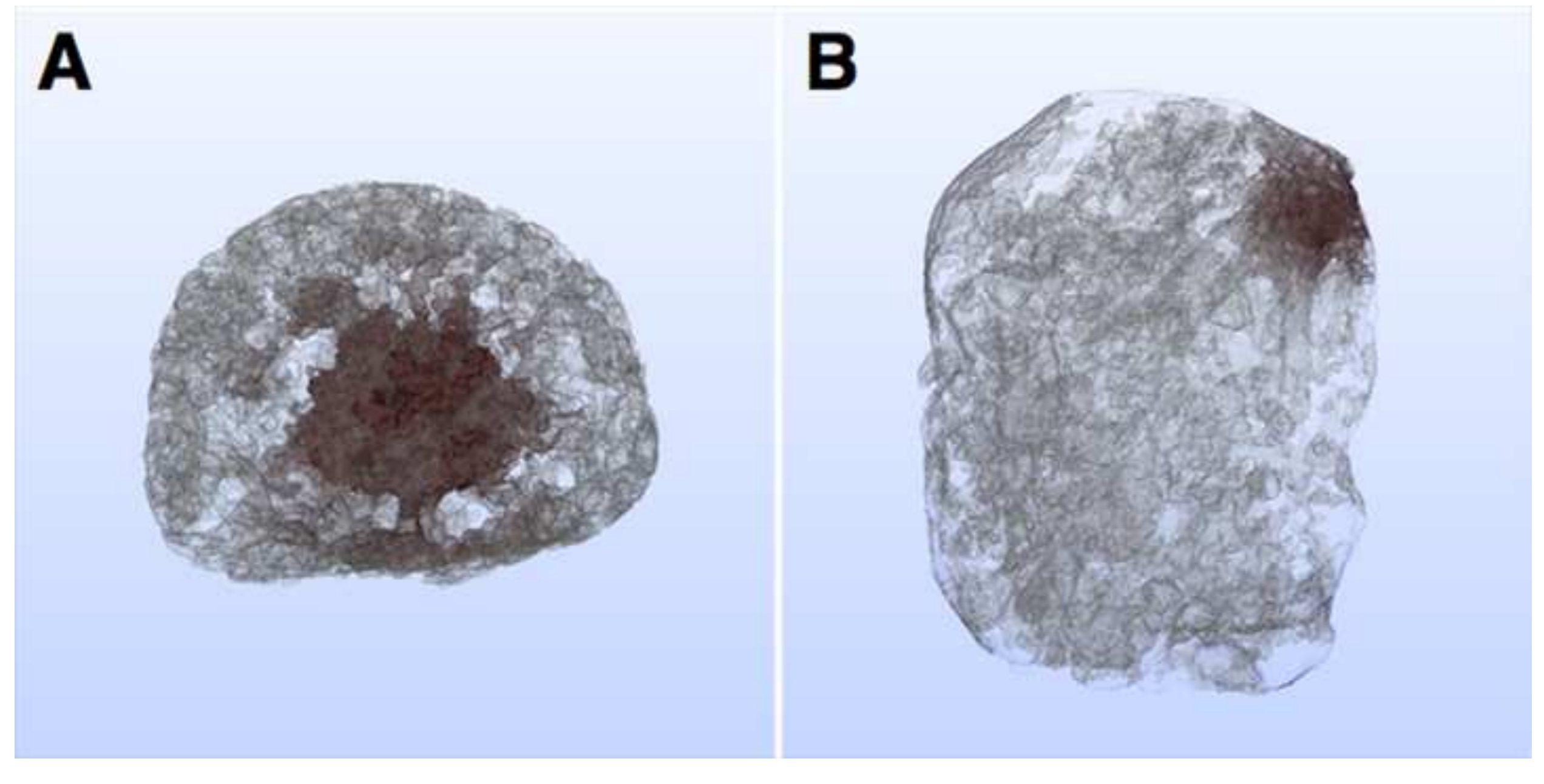

B
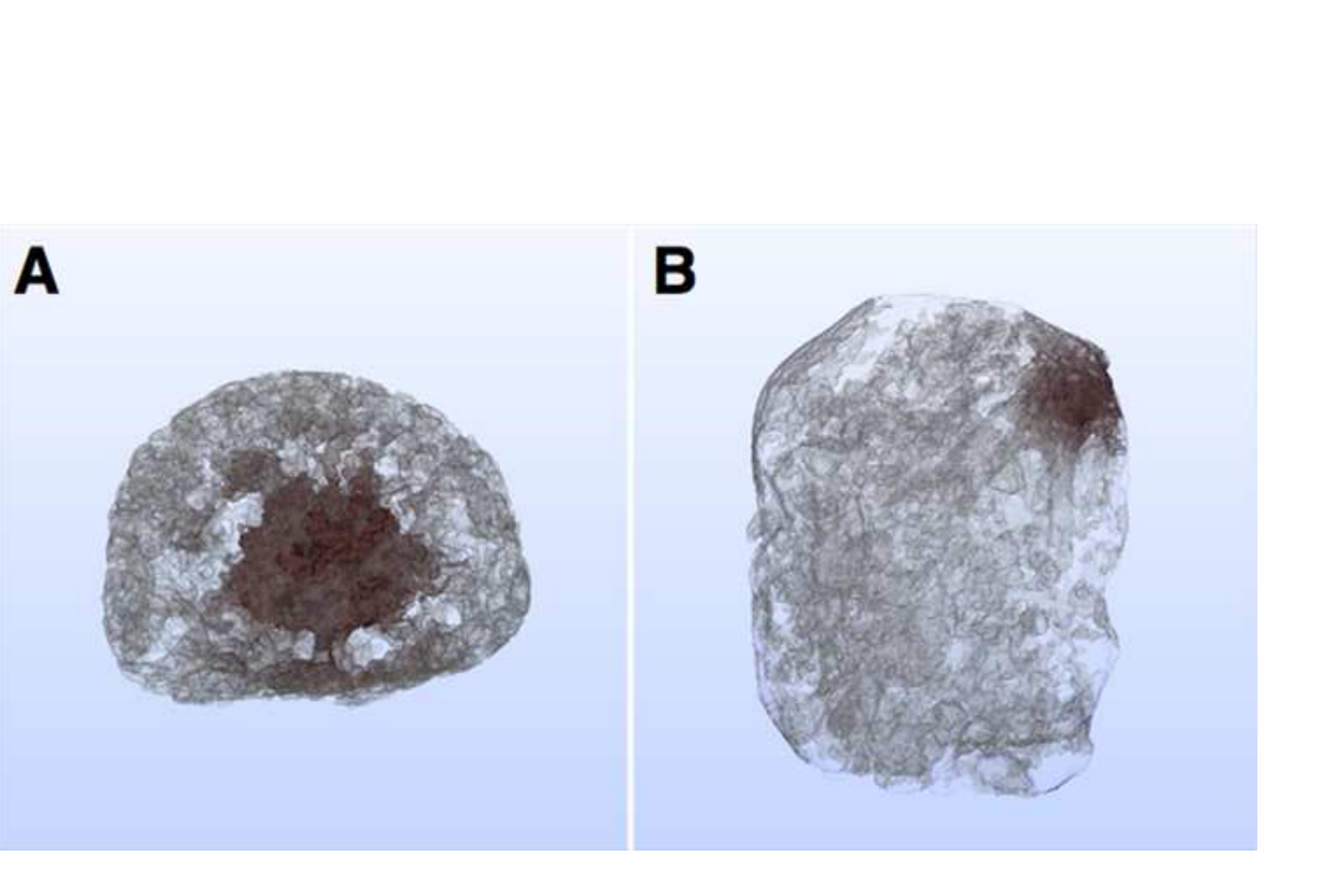

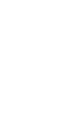



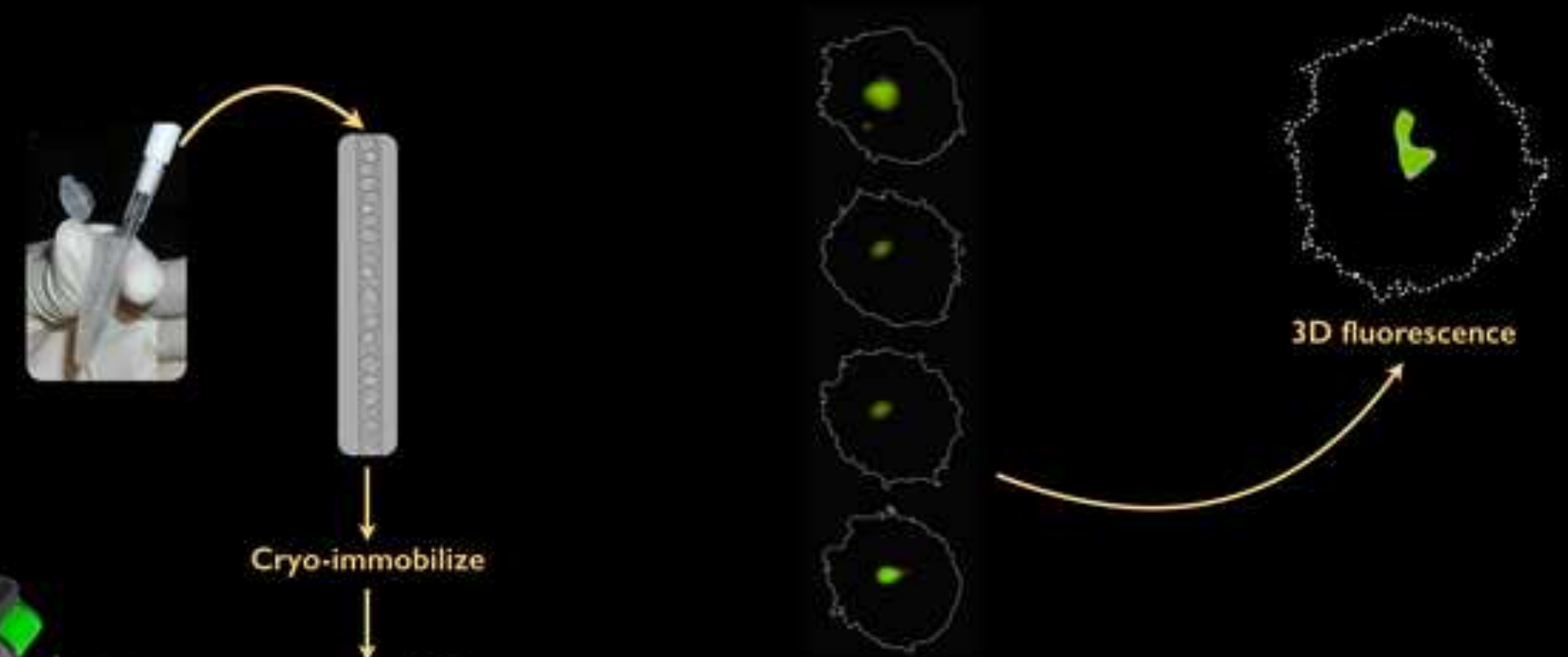

Optical sections
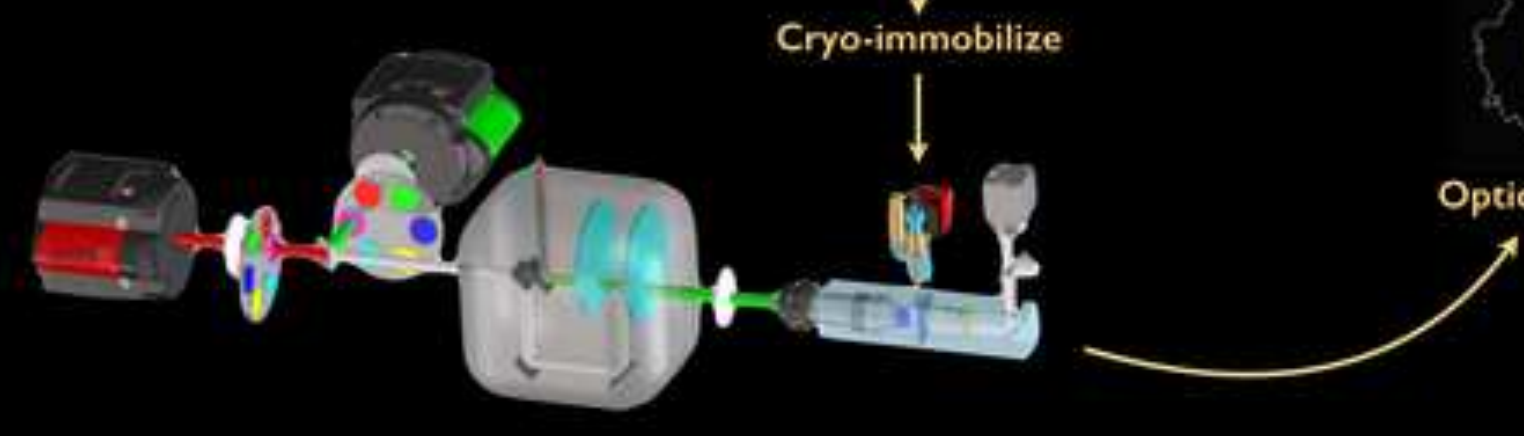

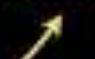


Cells in

Capillary

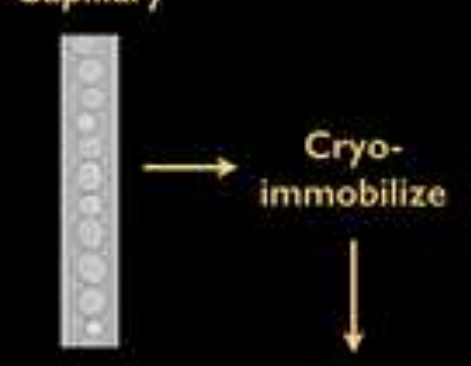

Cryo fluorescence
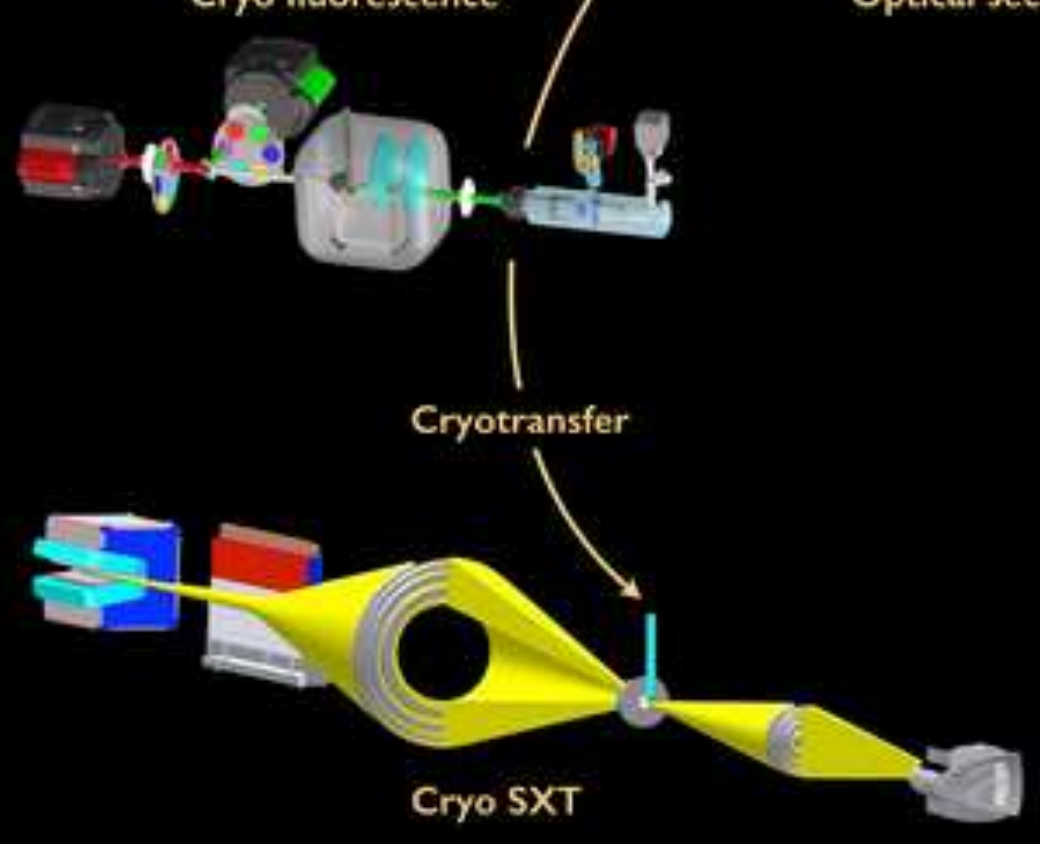

Optical section

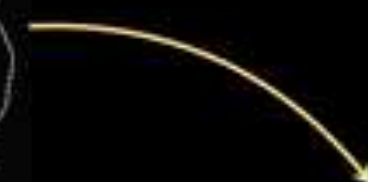

Segmentation

$>$
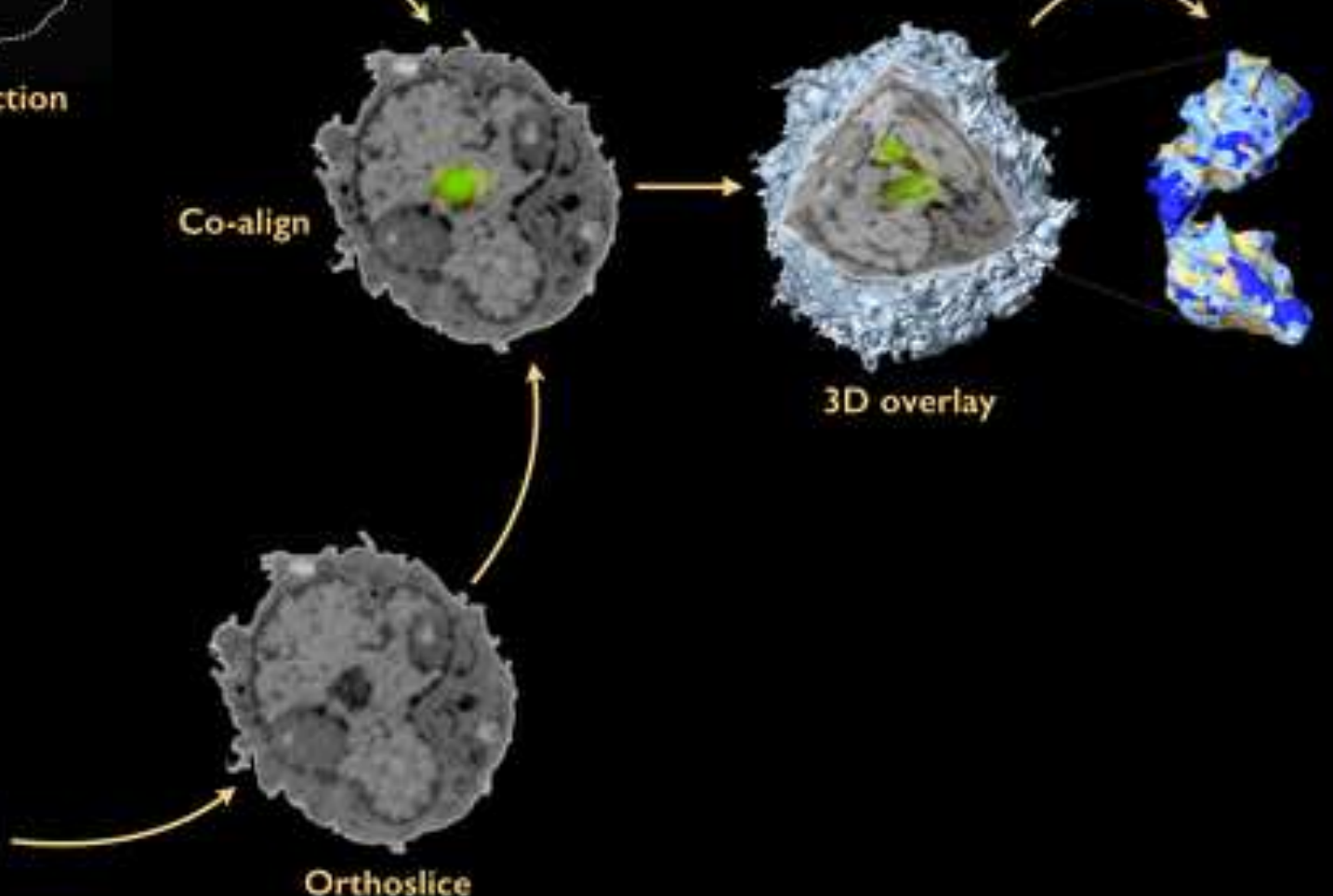

3D overlay 
Capillary decorated

with fiducial markers

Cryo-Fluorescence
Tomography
Soft X-ray

Tomography

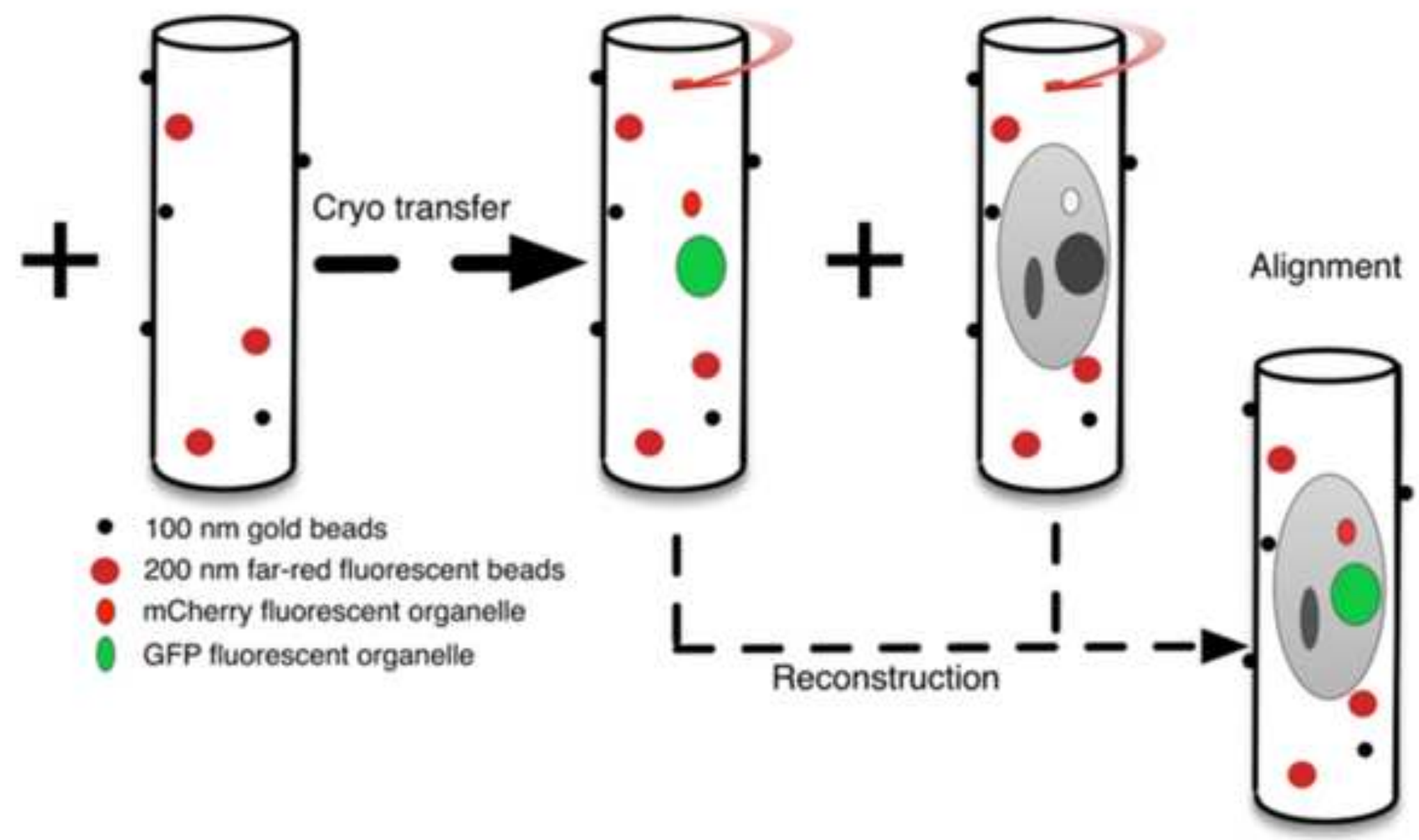



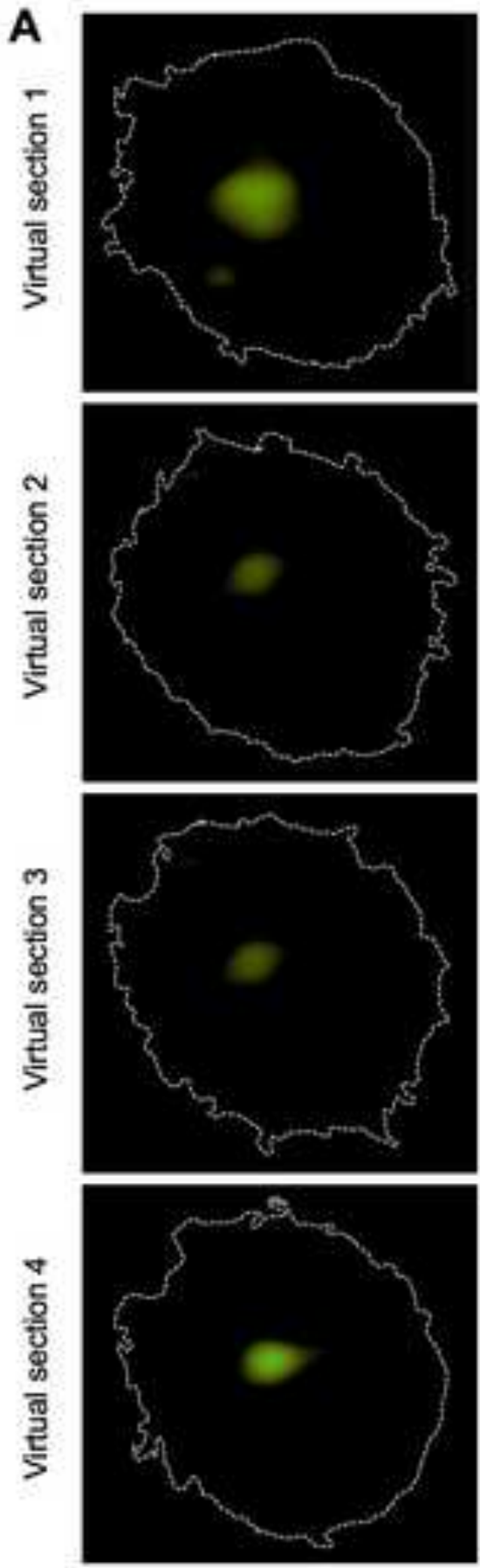

Figure

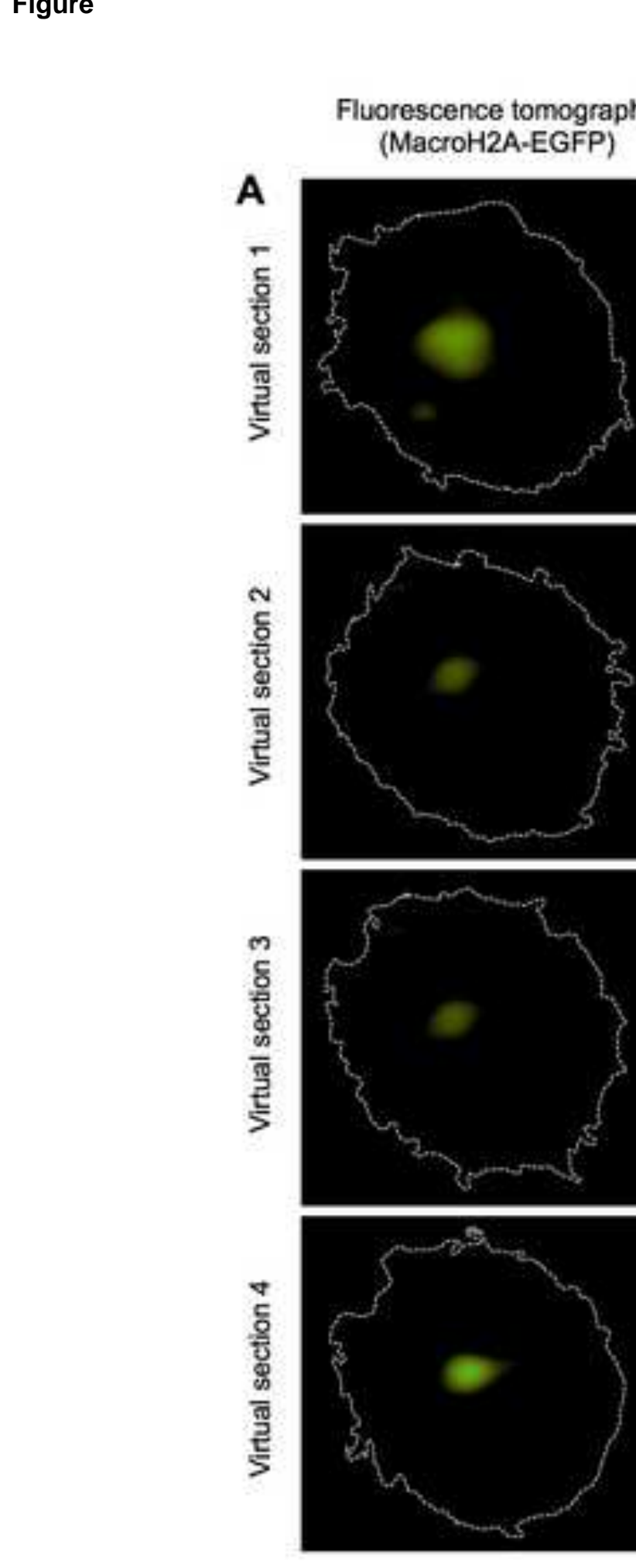

3D MacroH2A-EGFP 3D SXT cutaway

Soft x-ray tomography
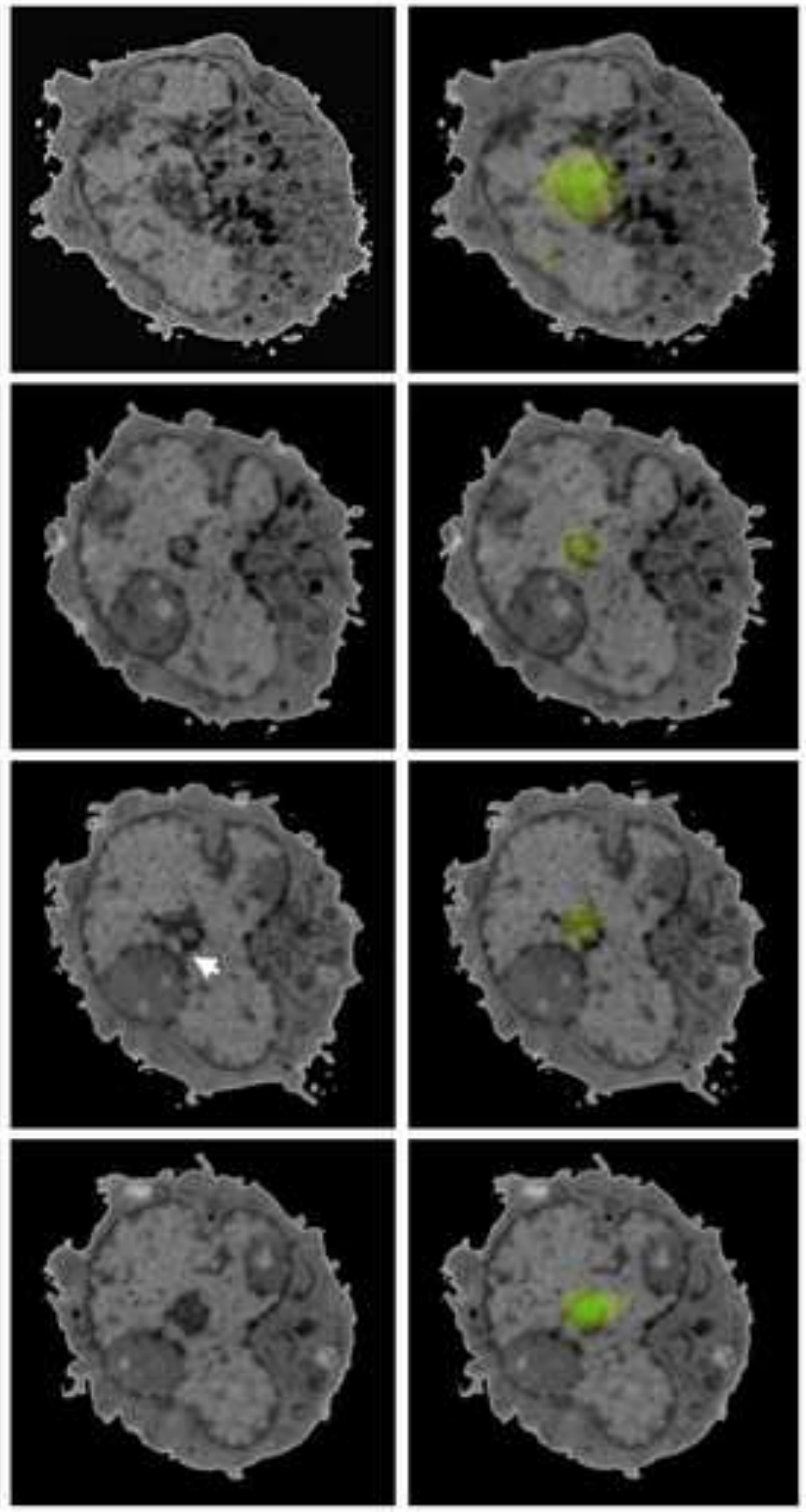

3D MacroH2A-EGFP 3D SXT cutaway Fluorescence
and SXT

Segmented Xi
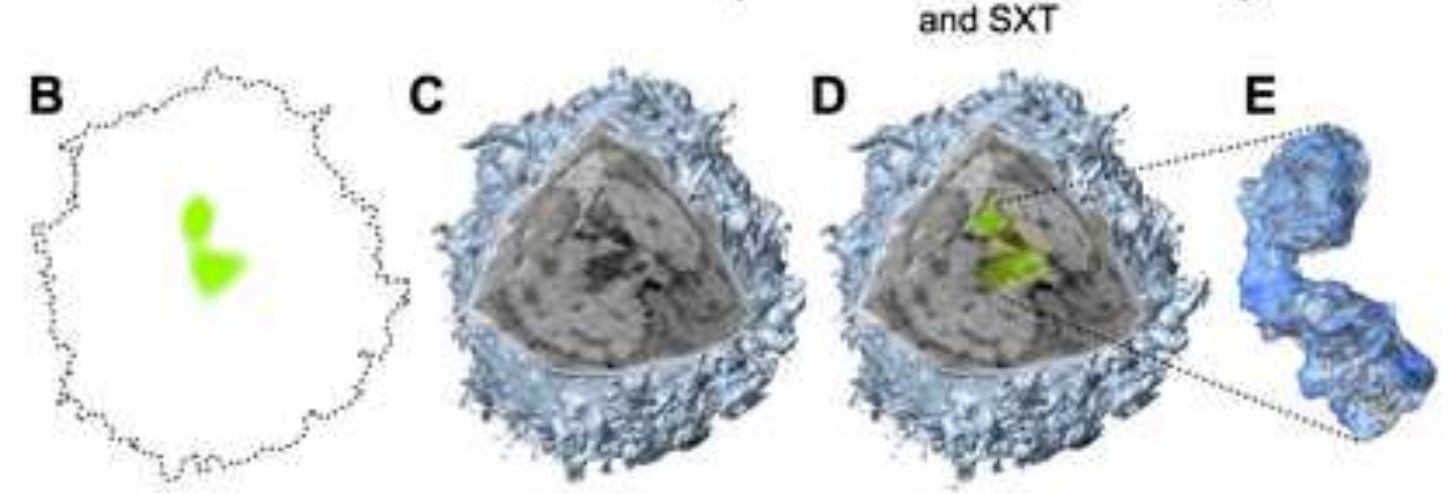

Overlay of fluorescence on $\mathrm{x}$-ray tomography
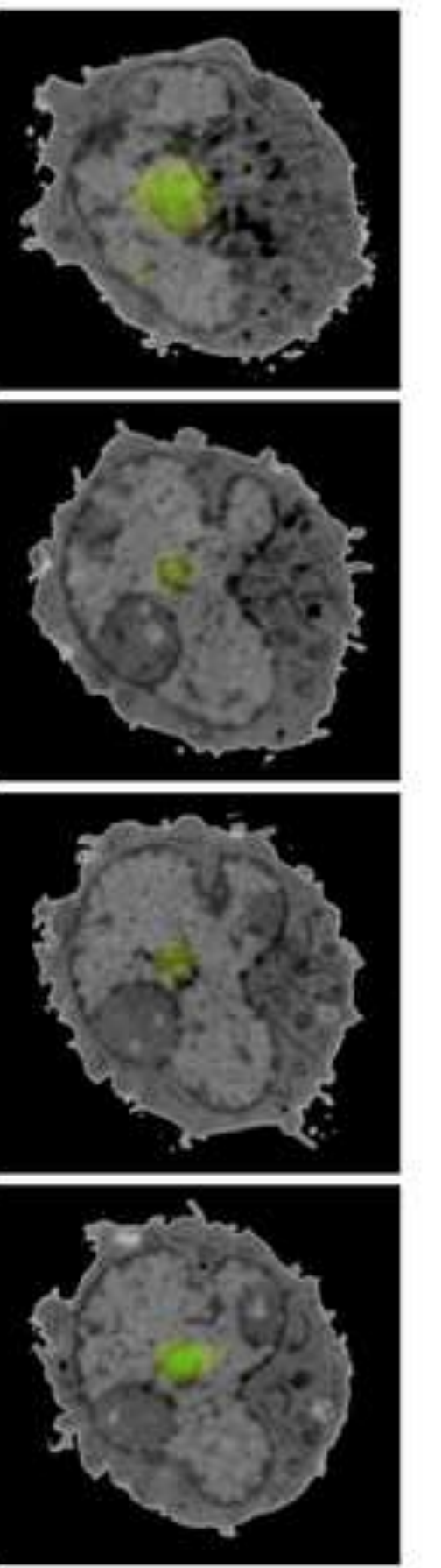

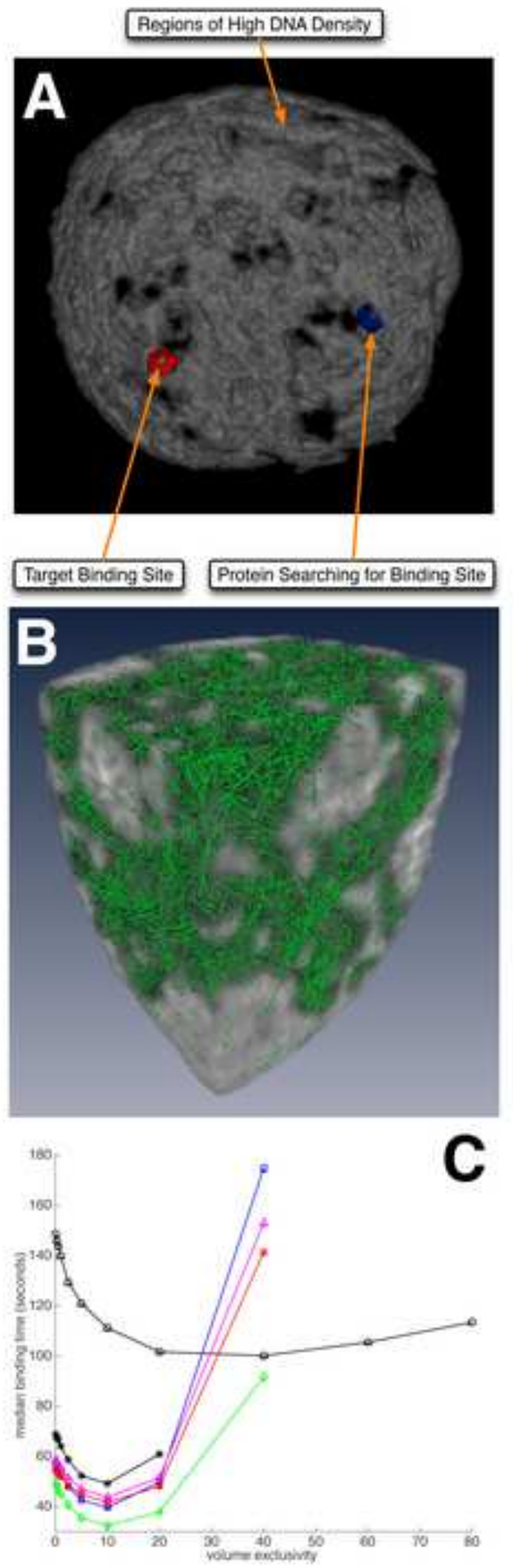
